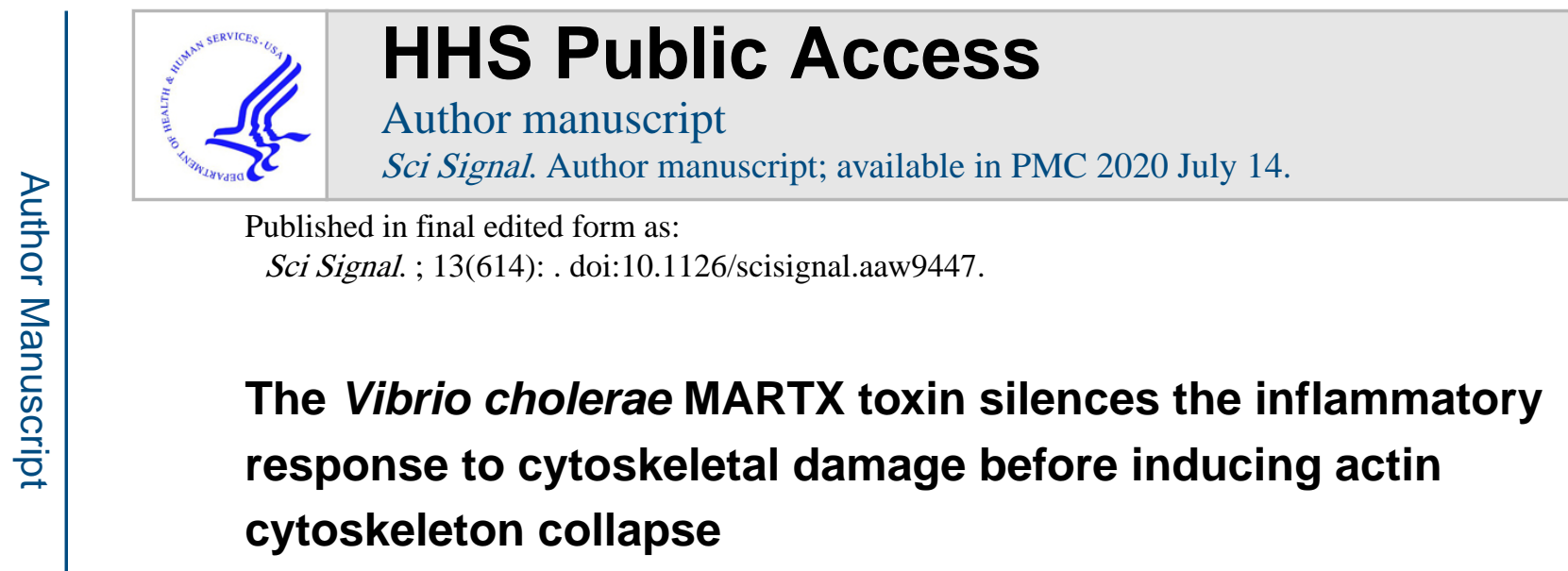

\author{
Patrick J. Woida ${ }^{1}$, Karla J. F. Satchell, ${ }^{1}$ \\ ${ }^{1}$ Department of Microbiology-Immunology, Northwestern University, Feinberg School of Medicine, \\ Chicago, IL 60611, USA
}

\begin{abstract}
Multifunctional autoprocessing repeats-in-toxin (MARTX) toxins are pore-forming bacterial toxins that translocate multiple functionally independent effector domains into a target eukaryotic cell. Vibrio cholerae colonizes intestinal epithelial cells (IECs) and utilizes a MARTX toxin with three effector domains - an actin cross-linking domain (ACD), a Rho inactivation domain (RID), and an $\alpha / \beta$ hydrolase domain $(\mathrm{ABH})$ - to suppress innate immunity and enhance colonization. We investigated whether these multiple catalytic enzymes delivered from a single toxin functioned in a coordinated manner to suppress intestinal innate immunity. Using cultured human IECs, we demonstrated that ACD-induced cytoskeletal collapse activated extracellular signal-regulated kinase (ERK), p38, and Jun N-terminal kinase (JNK) mitogen-activated protein kinase (MAPK) signaling to elicit a robust proinflammatory response characterized by the secretion of interleukin-8 (IL-8, also called CXCL8) and the expression of CXCL8, tumor necrosis factor $(T N F)$, and other proinflammatory genes. However, RID and ABH, which are naturally delivered along with ACD, blocked MAPK activation through Rac1 and thus prevented ACD-induced inflammation. RID also abolished IL-8 secretion induced by heat-killed bacteria, TNF, or latrunculin A. Thus, MARTX toxins utilize enzymatic multifunctionality to silence the host response to bacterial factors and to the damage caused by the toxins. Further, these data show how $V$. cholerae MARTX toxin suppresses intestinal inflammation and contributes to cholera being classically defined as a non-inflammatory diarrheal disease.
\end{abstract}

\title{
Editor's Summary
}

*Corresponding author. k-satchell@northwestern.edu.

Author contributions: P.J.W. conceptualized, designed, and conducted all experiments. K.J.F.S advised on all experiments. P.J.W. wrote the original draft of the manuscript and both P.J.W. and K.J.F.S. reviewed and edited the manuscript.

Competing interests: Dr. Satchell has a financial relationship with Situ Biosciences, LLC, a contract research organization with no relationship to her academic research program. Dr. Satchell holds patents and pending patents unrelated to research reported here. Dr. Satchell has accepted honoraria and travel support for public presentation of results reported here.

Data and material availability: Sharing of Vibro cholerae strains with academic researchers requires proof that the recipients have appropriate facilities and regulatory approval to work with pathogenic strains as well as a valid USDA or CDC permit. Requests for Vibro cholerae strains for commercial development or by non-academic researchers require individually negotiated MTAs. The RNAseq data have been deposited in the NCBI Gene Expression Omnibus (GEO), https://www.ncbi.nlm.nih.gov/geo/, under the accession number GSE125453. All other data needed to evaluate the conclusions in the paper are present in the paper or the Supplementary Materials. 
How Vibrio cholerae damages cells without triggering inflammation-The MARTX toxin of Vibrio cholerae (MARTX $_{V c}$ ) is a large protein that forms a pore in the plasma membrane and translocates multiple toxin effector domains into intestinal epithelial cells. These effectors include an actin cross-linking domain (ACD), a Rho inactivation domain (RID), and an $\alpha / \beta$ hydrolase domain $(\mathrm{ABH})$, as well as a protease that releases these effector domains into the host cytoplasm. Using human intestinal epithelial cells (IECs) in an in vitro model of infection, Woida and Satchell found that RID and ABH suppressed proinflammatory signaling that would otherwise have been activated by the cytoskeleton-damaging effects of ACD. RID and ABH also suppressed innate responses to pathogen-associated molecular patterns (PAMPs). These results reveal how the MARTX $_{V c}$ effector domains simultaneously promote virulence and suppress inflammatory responses and explain why cholera is a non-inflammatory disease.

One-Sentence Summary-The multifunctional MARTX toxin of Vibrio cholerae damages host cells and suppresses the innate response to that damage.

\section{INTRODUCTION}

Multifunctional autoprocessing repeats-in-toxin (MARTX) toxins incorporate multiple enzymatic functions to promote the virulence of various Vibrio species. MARTX toxins are secreted as single 3500 - 5300 amino acid (aa) polypeptides that contain conserved glycinerich repeats at the $\mathrm{N}$ - and $\mathrm{C}$-termini that flank multiple arrayed effector domains and an autoprocessing cysteine protease domain (CPD) (1). The glycine-rich repeats are proposed to form a pore in the plasma membrane of eukaryotic cells to translocate the arrayed effectors and the CPD into the target cell (2-4). In the cytoplasm, CPD is activated by binding to host inositol hexakisphosphate $\left(\mathrm{InsP}_{6}\right)$ and then auto-cleaves to free the effector domains from the large holotoxin. The individual effectors then traffic throughout the cell to identify targets and perform their catalytic functions (5-7) (Fig. 1A). Due to this enzymatic multifunctionality, MARTX toxins have been described as bacterial "cluster bombs" that release multiple cytotoxic bomblets into host cells from a single toxin warhead. Although the biochemical function of many of the effector domains is known (8), the additive or synergistic benefit of having all these enzymatic functions delivered on a single toxin has yet to be determined (9).

Vibrio cholerae is the causative agent of the severe diarrheal disease cholera (10). In addition to its primary virulence factor, the ADP-ribosylating cholera toxin, pandemic $V$. cholerae El Tor O1 strains secrete a 4,545 aa MARTX $V_{c}$ toxin that contributes to enhanced bacterial colonization of the small intestine by protecting the pathogen from neutrophil-mediated clearance during the earliest stages of infection (11-14). The early timing of these events suggests that the inhibition of neutrophils and other innate immune cells does not reflect destruction of the cells by the MARTX $V_{C}$ toxin, but rather a failure of neutrophils to be recruited to the site of infection. Therefore, the MARTX $_{V c}$ toxin might function to limit host signaling that results in innate immune cell recruitment.

Although MARTX toxins can have highly variable effector domain organizations in some species, the MARTX $V_{c}$ toxins of nearly all $V$. cholerae strains have the same three effector domains (15) (Fig. 1A). The first effector domain is the actin cross-linking domain (ACD) 
that covalently cross-links monomeric actin into oligomers that cannot be incorporated into microfilaments, thus disrupting the actin cytoskeleton (16-18). The cross-linked actin oligomers also have high binding affinity for formins and other actin-binding proteins, which further disrupts the cytoskeleton (19). The second domain is the Rho inactivation domain (RID) that inactivates Rac1 and other Rho-family GTPases by transferring fatty acids onto lysine residues in the C-terminal polybasic region of these GTPases. This acylation of the Rho GTPases blocks them from interacting with downstream effectors (20). The combined actions of ACD and RID results in destruction of the actin cytoskeleton and loss of epithelial cell junction integrity $(2,20,21)$. The final effector domain is the $\alpha / \beta$ hydrolase domain $(\mathrm{ABH})$, a highly specific phospholipase A1 that cleaves only phosphatidylinositol 3-phosphate (PI3P), thus inhibiting autophagy and endocytic trafficking (22). The activity of ABH also affects the activation state of the small GTPase CDC42, possibly due to the impact on the phosphorylation state of phosphatidylinositol lipids that control small GTPase activation as PI3P is depleted from the cell $(2,22)$. Each of the three effector domains can also individually disable macrophages in vitro (2), suggesting a mechanism by which the MARTX $V_{c}$ toxin protects against clearance of $V$. cholerae from the intestine. However, ACD alone potently inhibits phagocytosis, whereas inhibition by RID or $\mathrm{ABH}$ is far less robust (2). Therefore, in the context of co-introduction into cells in parallel with ACD, inhibition of phagocytosis is not likely the primary biological function of RID and $\mathrm{ABH}$ intoxication. Further, because the $\mathrm{MARTX}_{V c}$ toxin promotes colonization possibly even well before the onset of inflammation (11), inhibition of phagocytic clearance may not be a primary function of the MARTX $V_{V c}$ toxin.

Intestinal epithelial cells (IECs) act both as a barrier to contain bacteria in the gut lumen and as sensors to detect microbe-associated molecular patterns (MAMPs) and release chemokines that recruit immune cells to the site of infection $(23,24)$. Even though $V$. cholerae is typically considered a secretory, non-inflammatory diarrheal disease, these bacteria do elicit mild inflammation in the human intestine $(25,26)$. In addition, $V$. cholerae stimulates the release of the neutrophil-recruiting chemokine interleukin-8 (IL-8, also known as CXCL8) from human colonic intestinal cells in response to pathogen-associated molecular patterns (PAMPs), including purified flagellin and lipopolysaccharide (27-30). However, the extent to which $V$. cholerae induces IL-8 secretion in vitro in response to live bacteria varies dramatically based on the strain isolate $(27,28)$. We hypothesized that the variable response of IECs to live bacteria may reveal that $V$. cholerae actively suppresses proinflammatory signaling induced by PAMPs and that the variation among strain isolates is due to strain differences in secreted exotoxins.

In this study, we show that $V$. cholerae globally suppressed proinflammatory gene expression in IECs and that this signaling inhibition was mediated by the catalytic action of the secreted MARTX $V_{c}$ toxin, and not by other accessory toxins. These results explain observed differences in the inflammatory response to various $V$. cholerae strains and why infections with MARTX $V_{c}^{+} V$. cholerae induces a highly secretory, but non-inflammatory, diarrhea. Further, we found that the cytoskeletal destruction initiated by the ACD conversely functioned as a damage-associated molecular pattern (DAMP) that potently induced proinflammatory gene expression through the mitogen-activated protein kinase (MAPK) pathway even more robustly than did V. cholerae PAMPs. However, in the context of the 
MARTX $_{V c}$ holotoxin, co-delivery of RID and ABH silenced pathways that would normally transduce the signals to induce proinflammatory gene expression in response to both DAMPs and PAMPs. These data reveal that simultaneous delivery of all three effector domains on a single multifunctional toxin is advantageous because it enables the ACDmediated destruction of the actin cytoskeleton without detection of the associated damage by the infected host. Thus, multifunctional toxins can coordinate their multiple enzymatic activities to fine-tune the host response to infection. Further, this study supports that MARTX $_{V c}$ toxin suppression of inflammatory gene expression may reduce chemokine- and cytokine-dependent recruitment of immune cells to the site of infection and may thereby contribute to the differences in inflammation observed between various $V$. cholerae strains $(27,28)$.

\section{RESULTS}

\section{MARTX $_{V c}$, and not HlyA or HapA, inhibits IL-8 secretion from IECs}

$V$. cholerae secretes three accessory toxins in addition to cholera toxin: the MARTX $V_{c}$ toxin encoded by the gene $r t x A$, an a-hemolysin encoded by hlyA, and a hemagglutinin (HA)/ protease encoded by hap $A$. T84 human intestinal epithelial carcinoma cells have been previously used to characterize the intestinal innate immune response to $V$. cholerae and other enteric pathogens $(27,29,31)$. To evaluate whether accessory toxins modulated IL-8 secretion, we treated cultured T84 cells with live V. cholerae El Tor O1 strain N16961, which produces all three accessory toxins, or derivative strains with deletions in $r t x A, h l y A$, or hap $A$ (32) at a multiplicity of infection (m.o.i.) of 5. Bacteria were removed after 120 minutes ( $\mathrm{min}$ ), and IL-8 was assayed 20 hours later from media containing gentamicin. Whereas N16961 stimulated only small amounts of IL-8 to be secreted into the culture medium, similar to that secreted by untreated control cells (Fig. 1B), N16961 $\Delta r t x A$ induced significantly more IL-8 secretion (Fig. 1B). Bacteria lacking $h l y A$ or hap $A$, or both ( $\triangle$ hlyA $\triangle$ hapA, hereafter referred to as strain KFV119), did not stimulate IL-8 secretion (Fig. 1B). These results reveal that $V$. cholerae has the potential to stimulate the release of IL-8 from IECs, but the MARTX $V_{V}$ toxin actively suppressed the response.

The atypical El Tor O1 strain 2010EL-1786 from the 2010 Haiti outbreak is known to be more proinflammatory than other stains of $V$. cholerae (33). This strain has a premature stop codon in $r t x A$ that prevents secretion of the MARTX $V_{C}$ toxin (15). Consistent with results using N16961, 2010EL-1786 induced a significant increase in IL-8 secretion from IECs, but this response was suppressed when the premature stop codon in $r t x A$ was restored to a $\operatorname{Trp}$ codon (Fig. 1C). These results show that $V$. cholerae activated proinflammatory chemokine secretion that was suppressed in $r t x A^{+} V$. cholerae strains.

\section{MARTX $_{V c}$ toxin effector domains, and not the pore, inhibit IL-8 secretion in IECs}

The MARTX $\mathrm{V}_{\mathrm{V}}$ toxin has four putative cytotoxic functions: the formation of a pore and the biochemical activities of its three effector domains (9). In a previous study (2), we generated a derivative of KFV119 in which the $r t x A$ gene was modified to replace the sequences that encode the effector domains with an in-frame sequence for $\beta$-lactamase (Bla). This strain produces a toxin that forms a functional pore and is able to translocate Bla into the host cell, 
but lacks all effector activity (rtxA::bla). The individual effector domains ACD (acd::bla), RID (rid::bla), and ABH (abh::bla) were then individually restored into the parent $r t x A:: b l a$ strain to create toxins that delivered one of the three effectors (Fig. 2A-E). Strains expressing catalytically inactive derivatives of these effectors and a $\Delta r t x$ negative control strain were also generated (2). These strains were fully characterized, including demonstration of similar detection of Bla activity within eukaryotic cells after $60 \mathrm{~min}$ incubation of bacteria with epithelial cells across all strains (2).

The $r t x A::$ bla strain induced similar amounts of IL-8 secretion from IECs as did the $\Delta r t x$ strain, but significantly more IL-8 secretion than did KFV119 (Fig. 2F). This result indicates that the MARTX $V_{V c}$ pore alone does not suppress IL-8 secretion. Unexpectedly, the acd::bla strain induced significantly more IL-8 secretion compared to both the $\Delta r t x$ and $r t x A: \because b l a$ strains (Fig. 2F). The rid::bla strain induced IL-8 secretion equivalent to that induced by KFV119 (Fig. 2F). These results indicate that RID is sufficient to suppress $V$. choleraeinduced IL-8 secretion, whereas ACD actually exacerbates IL-8 secretion beyond that stimulated by live bacteria lacking the MARTX effectors (Fig. 2F). In fact, when IECs were simultaneously co-inoculated with both the acd::bla and the rid::bla strains at equal m.o.i of 2.5 (combined m.o.i of 5), IL-8 secretion was also suppressed (Fig. 2G). This was due to the acylation activity of RID, because the rid-H2782A.:bla catalytically inactive strain did not suppress IL-8 secretion. In addition, although less robust, co-inoculation of acd::bla with abh::bla also attenuated IL-8 secretion (Fig. 2G). These results reveal that RID, and to a lesser extent ABH, inhibited IL-8 secretion induced by ACD.

\section{The MARTX $V_{V c}$ toxin inhibits the expression of $C X C L 8$}

A block to IL-8 secretion can occur by inhibition of IL-8 protein secretion or inhibition of the expression of $C X C L 8$, which encodes IL-8. As done above, cells were treated with bacteria for $120 \mathrm{~min}$ and gene expression determined from cells collected from antibioticcontaining media. There was no significant change in the transcription of $C X C L 8$ in intestinal cells inoculated with KFV119 or $\Delta r t x$ compared to mock-treated cells (Fig. 2H) at two hours, when the bacteria were removed. There was also no significant change in cells exposed to bacteria producing only RID or ABH. By contrast, a significant 32-fold increase was induced by bacteria producing only ACD (Fig. $2 \mathrm{H}$ ). These results indicate that MARTX $_{V c}$ effector domain modulation of IL-8 secretion occurred primarily due to blocking of ACD-induced $C X C L 8$ gene transcription.

The absence of a response to $\Delta r t x$ was unexpected because $V$. cholerae alone induced IL-8 secretion (Fig. 1B and 2F) (27). Given that chemokine secretion from cells, as measured by ELISA, required a long incubation period following the $120 \mathrm{~min}$ bacterial challenge, we hypothesized that the IEC response to $V$. cholerae occurred after the exposure to bacteria, during the 20 hour incubation in antibiotic-containing media. Therefore, we measured changes in $C X C L 8$ expression over time following bacterial challenge. Confirming previous results, we observed no change in $C X C L 8$ expression by exposure to KFV119 at any timepoint measured after bacteria removal (Fig. 2I). However, although $\Delta r t x$ did not induce changes in expression at 120 min post-inoculation, there was a significant 10 -fold increase in expression four hours after bacterial removal (six hours post-inoculation) that eventually 
returned to baseline between 12 and 22 hours (Fig. 2I). As observed previously, acd::bla induced a significant 42 -fold change in $C X C L 8$ expression at two hours (Fig. 2I). Although this response diminished over time, the relative abundance of $C X C L 8$ mRNA remained 10to 18-fold higher than in mock-treated cells (Fig. 2I). These data further show that ACDinduced $C X C L 8$ expression was stimulated as much as four hours before $V$. choleraeinduced gene expression. Therefore, in addition to suppressing the host response to effector activity, $V$. cholerae may utilize the MARTX $V_{V c}$ toxin to disable host inflammatory responses prior to the detection of bacterial PAMPs, such as flagellin and lipopolysaccharide $(29,30)$.

\section{ACD, but not the MARTX $V_{V c}$ holotoxin, induces proinflammatory gene expression in IECs}

To determine the breadth of the proinflammatory immune response stimulated by ACD, we utilized a whole transcriptome RNA-sequencing (RNA-seq) approach. Cells were treated with various $V$. cholerae strains for two hours prior to transcriptional profiling. Statistically significant results were given a $-1 \leq 0 \leq 1 \log _{2}$ fold change cut off to adjust for biologically significant changes (Fig. $3 \mathrm{~A}$ and B, Table S1). A single gene $J U N$ was stimulated by KFV119 compared to uninoculated cells, but this stimulation was not consistent when validated by qPCR (Fig. 3C). Thus, there was no significant activation of any host gene after two hours treatment of IECs with MARTX $V_{c}+V$. cholerae.

Similarly, treatment of cells with $V$. cholerae rid::bla, abh::bla, or $\Delta r t x$ also did not induce significant changes in IEC gene expression by two hours. By contrast, the acd::bla strain induced differential regulation of over 200 genes (Table S2). Many of the genes significantly induced by ACD were identified as regulators of inflammation. These include genes encoding the chemokines CXCL8 (IL-8) and CXCL3 (IL-3), the cytokine tumor necrosis factor (TNF), and the proinflammatory transcription factors JUN, FOS, and EGR1, EGR2, and EGR3 (EGR1/2/3) (Fig 3B-G). The activation of select proinflammatory genes was validated by qPCR (Fig. 2H, 3C-G). These data suggest that, when delivered independently, ACD induced proinflammatory gene expression more rapidly than did $V$. cholerae alone (without ACD). However, when ACD was delivered as part of the complete MARTX $V_{c}$ toxin, the response was abolished.

\section{RID suppresses host MAPK signaling through Rac1 to modulate the ACD proinflammatory response}

Bioinformatic analysis of the genes differentially regulated genes by acd::bla revealed that many were associated with the extracellular signal-regulated kinase (ERK), p38, Jun Nterminal kinase (JNK) mitogen-activated protein kinase (MAPK) and nuclear factor $\mathrm{\kappa B}$ (NF$\kappa \mathrm{B})$ pathways (Fig. $3 \mathrm{H})$. These pathways have also been associated with the induction of IL-8 by purified $V$. cholerae flagellin (29). The phosphorylation of MAPK in bacteria-treated cells was monitored after two hours. Although KFV119-treated cells showed no ERK phosphorylation, $\Delta r t x$ activated ERK signaling (Fig. 3I). Further, acd::bla stimulated ERK phosphorylation above that of $\Delta r t x$ (Fig. 3I, Fig. S2A). Both the rid::bla and abh::bla strains suppressed ERK phosphorylation, whereas the rid-H2782A::bla and abh-H3369A::bla strains with catalytically inactive RID and ABH, respectively, did not (Fig. 3I, Fig S2A). KFV119-treated cells also elicited no phosphorylation of $\mathrm{p} 38$ or JNK. However, these MAPK pathways were activated by acd::bla (Fig. 3J and K, Fig. S2B and C). Although 
previous studies suggest that $V$. cholerae PAMPs may also activate these pathways (29), the observed limited or undetectable p38 and JNK MAPK phosphorylation by $\Delta r t x$ alone is likely a result of timing, because $V$. cholerae lacking toxins does not induce expression of proinflammatory genes until six hours post-inoculation (Fig. 2I and 3A). Finally, none of the effector domains inhibited NF- $\kappa$ B, as measured by I $\kappa$ Ba degradation (Fig. 3L, Fig. S2D).

Rac1 is a well characterized positive regulator of these pathways $(34,35)$. Whereas KFV119 suppressed Rac1 activation through the biochemical activity of RID, acd::bla significantly induced active GTP-bound Rac1 when compared to both PBS- and KFV119-treated cells (Fig. S1). In total, these data show that ACD should activate Rac1 to induce downstream MAPK. However, in the MARTX $V_{c}$ holotoxin, direct inactivation of Rac1 by RID blocked this Rac1-dependent signaling and thus blocked all MAPK signaling.

\section{RID and ABH inhibit ACD-induced MAPK signaling when stoichiometrically delivered from the same holotoxin}

The lack of MAPK activation in MARTX $V_{V c}^{+}$KFV119-treated cells suggests that there is active suppression of ACD-induced MAPK signaling due to interplay between the MARTX $_{V c}$ effector domains. However, these experiments rely on comparing strains with only one effector to those with either all or no effectors. Even though all Bla strains are known to deliver equivalent Bla activity (2), differences in IL-8 secretion across strains could result from minor variations in toxin expression, production, or effector delivery, particularly during co-inoculation studies (Fig. 2G). To address this potential problem, we generated six new strains in which single codons in $r t x A$ were changed to produce MARTX $_{V c}$ toxins that retained natural processing and stoichiometrically equivalent delivery of all effectors, but with one, two, or all three of the effectors carrying point mutations in essential catalytic site residues (Fig. 4A and Fig. S3A-H). Thus, each experiment with these strains was internally controlled for toxin expression and natural delivery of all three effectors at 1:1:1. All $\mathrm{ACD}^{+}$strains showed similar actin laddering patterns, indicative of equivalent ACD effector delivery (Fig. S4A and B).

These strains were then tested to determine the impact of different effector domain activities on IL-8 secretion when simultaneously delivered from the same toxin. These studies confirmed the above results with Bla strains that ACD alone induced IL-8 secretion, and that this activation was potently suppressed by RID (Fig. 4B). The amount of IL-8 secreted was equivalent between KFV119 and the ACD+RID strain with both active ACD and active RID but no active $\mathrm{ABH}$, demonstrating that RID dominates the inhibition of this pathway, independent of the presence of active $\mathrm{ABH}$.

ABH also suppressed ACD-induced IL-8 secretion when introduced into cells from the same toxin from the $\mathrm{ACD}+\mathrm{ABH}$ strain (Fig. 4B) with inhibition varying across experiments from 20-50\% (Fig. 4B and S5A-E). Thus, we considered that ABH might also inhibit Rac1 activation. As seen with the Bla strains, RID potently inhibited Rac1 activation compared to the Triple* strain in which all 3 effectors contained catalytically-inactivating substitutions and compared to the active ACD only strain (Fig. 4C). Although showing a lesser effect than RID alone, the ACD+ABH strain, in which both $\mathrm{ACD}$ and $\mathrm{ABH}$ are active, also reduced Rac1 activation compared to the active ACD-only strain, and this inactivation varied from 
20-50\% across independent experiments (Fig. 4C and 5S). The variable and more modest response supports that $\mathrm{ABH}$ inhibition of Rac1 was most likely indirect, which may explain the observed varying degree to which ABH suppressed both Rac1 activation and IL-8 secretion across independent experiments (Fig. 5SA-E), and why, unlike RID, ABH did not completely abolish IL-8 secretion (Fig. 2F and 4B).

To confirm that both RID and ABH suppressed the proinflammatory response to ACD through the inhibition of MAPK signaling, we examined the activation of these pathways in cells treated with the newly constructed strains. The active ACD only strain stimulated ERK activation beyond that of $V$. cholerae Triple* strain with no active effectors, whereas both the single active RID only and active ABH only strains suppressed bacterial activation of ERK (Fig. 4D, Fig. S6A). Additionally, both the active ACD+RID and active ACD+ABH strains showed attenuation of ACD-induced ERK phosphorylation, JNK phosphorylation, and p38 phosphorylation compared to the active ACD-only strain (Fig. 4D-F, Fig. S6A-C). Further, cells treated with the ERK inhibitor PD98059 showed no attenuation of the IL-8 response (Fig. 4G). However, inhibition of the p38 MAPK pathway by SB202190 inhibited IL-8 secretion (Fig. 4H), whereas inhibition of the JNK pathway by SP600125 had no effect on IL-8 secretion (Fig. 4I). In total, these data show that RID- and ABH-mediated suppression of Rac1 and MAPK signaling pathways, particularly the p38 pathway, attenuated the ACDinduced proinflammatory response.

\section{Cytoskeletal collapse activates proinflammatory signaling in ACD-treated cells in the absence of RID and ABH}

ACD has two putative cytotoxic functions. The first is the inhibitory action of toxic actin oligomers (dimers and trimers) that are formed when only $2-4 \%$ of total actin has been cross-linked $(19,36)$. The second is that ACD will eventually cross-link nearly $100 \%$ of cellular actin into higher order oligomers (10 to 15-mers), which sequesters bulk actin and induces cytoskeletal collapse (16). Kinetic experiments comparing the amount of actin cross-linking to MAPK activation were performed to determine which of these functions activate MAPK signaling. The $V$. cholerae strain with active ACD only cross-linked a maximum of $4 \%$ of total actin between 5 and 15 min after addition of the bacteria, corresponding to the formation of early toxic actin oligomers (Fig. 5A and B and Fig. S7AC). Cross-linking increased around $60 \mathrm{~min}$ (Fig. 5A and B), corresponding to ACD sequestering bulk actin to induce actin depolymerization and cytoskeletal collapse. In the same samples, MAPK signaling was activated between 60 and 90 min (Fig. 5C-H, Fig. S8A-B), when over 50\% total monomeric actin was cross-linked. These results indicate that a large portion of total actin must be cross-linked to activate proinflammatory signaling. Whereas cells exposed to the Triple* strain showed stochastic activation of MAPK signaling between 5 and $15 \mathrm{~min}$, they had significantly less ERK phosphorylation than cells exposed to the strain with active ACD only and no detectable phosphorylation of p38 or JNK between 30 and $120 \mathrm{~min}$ (Fig. S8C-H). This stochastic activation by the bacteria alone could account for the transient activation of $\mathrm{p} 38$ phosphorylation at $10 \mathrm{~min}$ that was observed in one experiment (Fig. S8A and S8B). These data suggest that it is ACD sequestration of bulk actin and cytoskeletal destruction, rather than the formation of toxic actin oligomers, that leads to the activation of proinflammatory signaling in IECs. 
To completely block the intestinal inflammatory response, RID and ABH would have to inactivate MAPK signaling prior to host detection of ACD-induced cytoskeletal collapse. This would indicate that instead of reversing the activation state of MAPK pathways after 60-90 min, RID and ABH inactive these pathways ahead of ACD-induced MAPK activation. Treating cells with KFV119 failed to induce any changes in MAPK activation even though it induced actin cross-linking (Fig. 6A-F). Further, cells exposed to the ACD +RID strain or the ACD+ABH strain showed reduced phosphorylation of ERK, p38, and JNK compared to KFV119-treated cells, even though there was actin cross-linking at 60 min (Fig. S9A-L). Additionally, MAPK activation induced by the ACD+RID and ACD+ABH strains was still significantly less than activation by the strain with only active ACD at 120 min (Fig. 6G-I). These data suggest that RID and ABH were each sufficient to attenuate ACD-mediated induction of MAPK signaling. However, modest, yet significant, activation of the JNK pathway by the ACD+RID strain was still observed at 120 min (Fig. S9A-F). The ACD+ABH strain also induced slight, yet significant, activation of the p38 and JNK pathways at 120 min (Fig. S9G-L). Given that both RID and ABH alone did not completely abolish activation of these pathways, both effectors may be required to completely abolish the global inflammatory response observed in the RNA-seq analysis (Fig. 3A). The inhibition of MAPK signaling was not due to RID and ABH directly inhibiting ACD actin cross-linking activity, because the ACD+ABH strain showed a slight, yet significant, increase in actin cross-linking at 30 and 60 min before returning to similar amounts at 90 and 120 min (Fig. S9M). Therefore, both RID and ABH independently silenced signal transduction pathways prior to host detection of ACD-induced collapse of the actin cytoskeleton without modulating ACD activity.

\section{RID inhibits IL-8 secretion induced by ACD- or latrunculin A-mediated actin destruction}

RID was more potent than $\mathrm{ABH}$ in inhibiting the $\mathrm{ACD}$-induced inflammatory response (Figs. 2F, 2G, 4B, and 4C). To determine whether the anti-inflammatory action of RID was specific to MARTX-intoxicated cells or also occurred independently of MARTX, cells were treated with purified recombinant RID or ACD fused to the N-terminal domain of the lethal factor (LF) component of anthrax toxin ( $\mathrm{LF}_{N} \mathrm{RID}$ and $\mathrm{LF}_{\mathrm{N}} \mathrm{ACD}$, respectively) in combination with the anthrax toxin component protective antigen (PA), which mediates entry of LF into cells. This previously characterized system allows for the delivery of MARTX effector domains into cells in the absence of bacteria or MARTX holotoxin (Fig. 7A) (37). $\mathrm{LF}_{N} \mathrm{RID}$ has been demonstrated to acylate Rac1 in cells (20), and $\mathrm{LF}_{N} \mathrm{ACD}$ is sufficient for actin cross-linking (37). Treating cells with $\mathrm{LF}_{\mathrm{N}} \mathrm{ACD}$ and PA induced IL-8 secretion, demonstrating that $\mathrm{ACD}$ was sufficient for induction of a proinflammatory immune response in the absence of a bacterium (Fig. 7B). This inflammatory response was not limited to actin cytoskeleton damage due to the toxic action of $\mathrm{ACD}$, because treating cells treated with latrunculin A, a sponge toxin that binds and sequesters G-actin, also induced IL-8 secretion. Treatment of cells with $L_{N} R I D$ in the presence of PA suppressed latrunculin A-induced IL-8 secretion (Fig. 7C). In contrast, inhibiting formins with SMIFH2, which affects actin cytoskeleton remodeling without directly causing cytoskeletal collapse, failed to elicit an IL-8 response (Fig. 7D). These data support that it is the onset of cytoskeletal collapse, rather than limited cytoskeletal damage that stimulates the 
inflammatory pathways and that, regardless of the inducer, RID inhibits activation of these pathways.

RID's inhibition of the proinflammatory immune response to bacterial factors also extended to inflammation induced by other stimuli. Treatment of cells with LF $_{\mathrm{N}} \mathrm{RID}$ and PA was also sufficient to suppress IL-8 secretion induced by heat-killed bacteria (Fig. 7E) and to attenuate IL-8 secretion in response to purified TNF (Fig. 7F). The residual stimulation of IL- 8 by TNF most likely occurred through TNF induction of NF- $\kappa$ B signaling, which RID did not inhibit (Fig. 3L). In total, these data demonstrate that RID potently inhibited inflammation induced by PAMPs and by distinct pathways of cytoskeletal damage, indicating that RID is also a general inhibitor of actin cytoskeleton damage-associated molecular patterns (DAMPs) (Fig. 8A-C).

\section{DISCUSSION}

MARTX toxins are unique hybrids of independently secreted toxins and multifunctional effector delivery systems $(8,38)$. This study demonstrates that the $V$. cholerae MARTX toxin utilizes this multifunctionality to "self-regulate" and silence the host response both to its own cytotoxic activity and to the detection of bacterial PAMPs. Following translocation into the host cell, ACD begins cross-linking actin to produce early toxic oligomers that sequester actin-binding proteins and disrupt intestinal tight junctions. While these toxic oligomers are being formed, RID directly inactivates Rac1, and ABH cleaves PI3P to directly and indirectly prevent Rac1 activation, respectively. At this early timepoint, the MAPK signaling pathways that trigger inflammatory responses to DAMPs are not activated. Once ACD begins to sequester bulk actin and induce cytoskeletal collapse, the cell is unable to trigger Rac1 and MAPK activation. Therefore, the MARTX $V_{\mathcal{C}}$ toxin blocks IECs from activating an innate immune response (Fig. 8A-C). Although previous studies have suggested that MARTX $^{+} V$. cholerae strains induce IL-8 secretion, those studies found the proinflammatory response of epithelial cells to depend on the growth phase of the bacteria because stationary phase cultures elicited more IL- 8 production than did log phase cultures (27). Secretion of the MARTX $_{V c}$ toxin is also growth phase-dependent; it is produced and secreted during log phase, and then toxin that is present in the supernatant is degraded by proteases that are secreted during stationary phase (39). Therefore, no toxin would be present to suppress IL-8 secretion in stationary-phase culture fluids. Our findings support a new model in which MARTX $_{V c}$ toxin produced during the early active growth stage in the intestines acts to suppress the IEC inflammatory response. These data indicate that MARTX $V_{c}$ toxin suppression of intestinal innate immunity prevents host recruitment of immune cells to protect the colonizing bacteria from neutrophil-mediated clearance.

Additionally, MARTX $V_{c}$ toxin immunomodulatory activities may contribute to the differences in inflammation observed between various $V$. cholerae strains $(27,28)$. In particular, the current predominant circulating "altered" El Tor V. cholerae strains responsible for pandemic disease since the mid 2000's are hypervirulent and exhibit increased clinical severity of diarrhea $(40,41)$. This is in part due to the acquisition of mutations in the genes encoding the histone-like nucleoid staining protein (H-NS) and the two-component system response regulator VieA, resulting in increased production of cholera 
toxin and HlyA, increased bacterial motility, and greater inflammasome activation, suggesting increased inflammation $(33,41,42)$. In addition, these strains naturally lack the MARTX $_{V c}$ toxin due to a stop codon in $r t x A(15)$. Restoring toxin secretion in altered El Tor isolate 2010EL-1786 attenuated IL-8 induction comparable to the parent strain (Fig. 1C). Thus, loss of the MARTX $V_{c}$ toxin immunomodulatory activities in these altered strains may increase intestinal inflammation, which could exacerbate disease severity and contribute to its hypervirulence.

The effector domains, and not the MARTX $_{V c}$ pore, were found to modulate innate immune signaling in IECs. These data support a previously established model in which the MARTX pore functions primarily as an effector delivery platform and not a direct virulence mechanism (43). How each delivered effector contributes to pathogenesis is still debated. Although other studies have connected RID and ABH to inhibition of macrophage phagocytosis in vitro $(20,44)$, these conclusions contradict previous findings that the $5 \%$ inhibition of phagocytosis by RID and $\mathrm{ABH}$ is biologically insignificant compared to the 90\% inhibition by ACD (2). Our study supports the hypothesis that RID and ABH most likely function primarily to abolish host detection of cytoskeletal damage (a DAMP) and bacterial PAMPs. RID-mediated inactivation of MAPK pathways most likely occurs through its direct inactivation of Rac1 and direct or indirect inactivation other Rho family GTPases, thereby blocking responses to ACD-induced cytoskeletal damage. Rho-family GTPases are known to both positively and negatively regulate MAPK and other cell signaling pathways, and inactivation of them by bacterial toxins can inhibit these pathways in specific cell types $(34,35)$. However, toxin-mediated inactivation of Rho GTPases through covalent modifications, such as ampylation or glycosylation, induces IL-8 secretion in IECs and activates the pyrin inflammasome in macrophages $(45,46)$. However, RID does not activate the inflammasome (46). Therefore, RID acylation of the C-terminal polybasic region of Rho family GTPases allows for the inactivation of Rho GTPases to suppress proinflammatory signaling pathways in IECs, while also evading host detection of Rho inactivation through the inflammasome.

How cleavage of PI3P by ABH blocks Rac1 activation and proinflammatory signaling is still unknown. Some Rho-family GTPases utilize guanine nucleotide exchange factors (GEFs) whose activity is regulated by phosphatidylinositol binding to promote activation of Rho GTPases (47). For example, the C-terminal domain of the Rac1 GEF Tiam-1 has weak binding affinity for PI3P. Although this activity is not required for Tiam-1 subcellular localization, abolishing Tiam-1 PI3P binding inhibits Rac1 activation (48). ABH depletion of PI3P may also disrupt the equilibrium of other phosphatidylinositol lipids, such phosphatidylinositol $(3,4,5)$ triphosphate (PIP3) or phosphatidylinositol $(3,4)$ diphosphate (PIP2), both of which spatially regulate Rac1 GEFs and GTPases activating proteins (GAPs) and control formation of active Rac1 nanoclusters in membranes (49, 50). Additionally, PI3P is required for the formation of autophagosomes, and $\mathrm{ABH}-$ mediated cleavage of PI3P inhibits the formation of autophagosomes and endocytic trafficking (22). ABH depletion of PI3P could also block the formation of scaffolding complexes on autophagosomes and endosomes that promote recruitment and activation of MAPK signaling molecules $(51,52)$. The loss of the scaffolds would further prevent activation of the MAPK pathway. 
ACD production of toxic actin oligomers has been characterized as the primary cytotoxic mechanism of ACD based on the hypothesis that the kinetics of ACD actin cross-linking prevent it from cross-linking the majority of cellular actin (19). Our study reveals that ACD stimulation of MAPK signaling pathways occurred after cross-linking of over $50 \%$ of cellular actin (Fig. 6,7). This supports previous findings in which type VI secreted ACD cross-links significant quantities of actin and induces intestinal inflammation in mice (53). Although the toxic oligomers may still contribute to cytoskeletal collapse, our data indicate that it is the destruction of the cytoskeleton, and not the inhibition of formins by toxic actin oligomers, that activates proinflammatory signaling.

Synergistic and antagonistic interactions between co-delivered bacterial effectors can have a profound impact on the host response and virulence. The Type III secreted effectors SopE and SopB from Salmonella enterica serovar Typhimurium work in synergy to recruit myosin VI to the plasma membrane to facilitate actin rearrangement and promote bacterial invasion (54). Other combinations of $S$. enterica Type III secretion effectors have also been shown to display synergistic or antagonistic interactions (55). YopE and YopT from Yersinia pestis independently activate the inflammasome; however, inflammasome activation is suppressed during infection by the co-delivered effector YopM, and $Y$. pestis strains lacking YopM are less virulent in mice due to the immune response from the inflammasome (56). Similarly, RID and $\mathrm{ABH}$ silencing the global response to ACD activity provide evidence that different MARTX effector domain combinations could impact the overall host response to codelivered effectors. Effector translocation from the same toxin assures stoichiometric delivery that can further maximize the synergistic benefit. These data suggest that an effector's contribution to virulence and disease could be enhanced or attenuated depending on which other effector domains are delivered with it. Vibrio vulnificus, for example, has different natural effector combinations that confer variations in virulence potential and promote distinct host responses (57-59). Therefore, MARTX toxin multifunctionality, in combination with variability in the effector domain repertoire, allows for variations in effector combinations and interplay that could either enhance or attenuate MARTX toxinassociated virulence.

\section{MATERIALS AND METHODS}

\section{Antibodies and chemical reagents}

Antibodies used in this study include phospho-p44/p42 MAPK (ERK1/2) (Cell Signaling Technology, \#4337S), p44/p42 MAPK (ERK1/2) (Cell Signaling Technology, \#4965S), phospho-p38 MAPK (Cell Signaling Technology, \#9211S), p38 MAPK (Cell Signaling Technology, \#9212S), phospho-SAPK/JNK (Cell Signaling Technology, \#9255S), SAPK/JNK (Cell Signaling Technology, \#9252S), Vinculin (Cell Signaling Technology, \#13901), IKBa (Cell Signaling Technology, \#9242S), Tubulin (Cell Signaling Technology, \#2144), GAPDH (Santa Cruz, \#sc-25779), Actin (Sigma, \#A2066), and LICOR IRDye 800CW/680LT secondary antibodies (LICOR, \#926-3221, \#926-3211, \#926-68070, \#92668071). Chemical reagents used in this study were ERK MAPK inhibitor PD98059 (Cell Signaling Technology, \#9900L), p38 MAPK inhibitor SB202190 (Cell Signaling Technology, \#S7067), JNK MAPK inhibitor SP600125 (Abcam, \#120065), Latrunculin A 
(Millipore, \#428021), and SMIFH2 (Fisher Scientific, \#440110) and recombinant human TNF (R\&D Systems, \#210-TA-005/CF).

Cell culture-T84 male colorectal carcinoma cells acquired from the American Type Culture Collection (ATCC, \#CCL-248) were cultured in Dulbecco's Modified Eagle Medium/Nutrient Mixture F-12 (DMEM/F12), GlutaMAX supplement (ThermoFisher Gibco, \#10565018) with 10\% (w/v) fetal bovine serum (FBS, Gemini Bio-products, \#900108 ) and $0.1 \%$ penicillin/streptomycin (ThermoFisher Gibco). HeLa cells (ATCC, \#CCL-2) were grown in DMEM (ThermoFisher Gibco, \#11965) with 10\% FBS and 0.1\% penicillin/ streptomycin. Cells were maintained at $37^{\circ} \mathrm{C}$ in the presence of $5 \% \mathrm{CO}_{2}$.

Bacterial growth medium-Bacterial strains and plasmids used in this study are listed in Table S3. All $V$. cholerae strains used in this study are derived from spontaneous streptomycin-resistant derivatives of clinical isolates N16961 or 2010EL-1796. V. cholerae and Escherichia coli were grown on Luria-Bertani (LB) broth or agar. $V$. cholerae medium was supplemented with $100 \mu \mathrm{g} \mathrm{mL}^{-1}$ streptomycin, $2 \mu \mathrm{g} \mathrm{mL}^{-1}$ chloramphenicol, $100 \mu \mathrm{g} \mathrm{mL}$ ${ }^{-1}$ ampicillin, or $5 \%(\mathrm{w} / \mathrm{v})$ sucrose as needed. E. coli growth medium was supplemented with $10 \mu \mathrm{g} \mathrm{mL}^{-1}$ chloramphenicol, $100 \mu \mathrm{g} \mathrm{mL}^{-1}$ ampicillin, or $50 \mu \mathrm{g} \mathrm{mL}^{-1}$ kanamycin as needed.

Treatment of cells with live bacteria- $V$. cholerae strains were grown at $30^{\circ} \mathrm{C}$ in $\mathrm{LB}$ medium supplemented with $100 \mu \mathrm{g} \mathrm{mL}^{-1}$ streptomycin. Overnight cultures were diluted $1: 100$ and grown at $30^{\circ} \mathrm{C}$ with shaking until exponential phase $\left(\mathrm{OD}_{600} \approx 0.40-0.60\right)$. Bacteria from $1 \mathrm{~mL}$ were pelleted by centrifugation and resuspended in phosphate buffered saline (PBS) to a final concentration of $5 \times 10^{8}$ bacterial cells $\mathrm{mL}^{-1}$. T84 cells (seeded the day before into cell culture-treated plates) were twice washed with PBS and then media changed to antibiotic- and FBS-free DMEM/F12. Resuspended $V$. cholerae was added to media over cells (multiplicity of infection $=5$ ). Inoculations were synchronized by centrifugation at 500 $\mathrm{x} g$ for $3 \mathrm{~min}$. T84 cells were subsequently incubated at $37^{\circ} \mathrm{C}$ in the presence of $5 \% \mathrm{CO}_{2}$ until processed for downstream applications.

$\mathbf{L F} \mathbf{F}_{\mathbf{N}}$ Effector intoxication of T84 cells- $-\mathrm{LF}_{\mathrm{N}}$ fusion proteins were expressed from plasmids pTCO24 (LF $\left.{ }_{\mathrm{N}} \mathrm{ACD}\right)$ and pKS119 (LF $\left.\mathrm{N}_{\mathrm{N}} \mathrm{RD}\right)$ and purified as previously described $(21,37)$. In brief, E. coli containing BL21(DE3)(pMagic) with overexpression plasmids were grown in Terrific broth and expression of protein induced with $1 \mathrm{mM}$ isopropyl $\beta$-Dthiogalactopyranoside at $25^{\circ} \mathrm{C}$ overnight. Bacteria were harvested in Buffer A (10mM Tris, $500 \mathrm{mM} \mathrm{NaCl} \mathrm{pH} 8.3$ ), lysed by sonication, and lysate clarified by centrifugation at 16,0000 $\mathrm{x} g$ for $30 \mathrm{~min}$. The $6 x$ His-tagged recombinant proteins were purified using Ni-NTA HisTrap column followed by size-exclusion chromatography using a Superdex 75 column in Buffer A with $5 \mathrm{mM} \beta$-mercaptoethanol using the ÄKTA protein purification system (GE Healthcare). Proteins were stored in $10 \%$ glycerol at $-80^{\circ} \mathrm{C}$.

For intoxication of cells, media was replaced over $10^{5}$ cells previously seeded in a 12 -well plate. $31.7 \mathrm{nM}$ PA alone (List Labs, \#171E) or in the presence of $13.6 \mathrm{nM} \mathrm{LF}_{\mathrm{N}}, \mathrm{LF}_{\mathrm{N}}-\mathrm{ACD}$, or $\mathrm{LF}_{\mathrm{N}}-\mathrm{RID}$ was added to media and cells were incubated for $20 \mathrm{hr}$ at $37^{\circ} \mathrm{C}$ in the presence of $5 \% \mathrm{CO}_{2}$. For co-intoxication of $13.6 \mathrm{nM} \mathrm{LF}_{\mathrm{N}} \mathrm{ACD}$ and $\mathrm{LF}_{\mathrm{N}}-\mathrm{RID}, 3 \mathrm{x}$ excess PA was used 
to ensure equal translocation of both effectors. Co-treatment of $1 \mu \mathrm{M}$ latrunculin $\mathrm{A}$ or 5 $\mathrm{ng} / \mathrm{mL}$ of recombinant TNF were used when indicated. For studies using co-treatment of boiled bacteria, $1 \mathrm{~mL}$ of an overnight culture of the $\Delta r t x$ was pelleted by centrifugation and resuspended in $1 \mathrm{~mL}$ of PBS. The resuspended bacteria were boiled for 10 minutes at $95^{\circ} \mathrm{C}$. $20 \mu \mathrm{L}$ of the boiled culture was used to stimulate IL- 8 secretion in T84 cells were indicated.

Construction of pDS132 sacB-counterselectable plasmids-Fragments (gBlocks) containing either the ACD E1990A mutation or the ABH H3369A mutation along with 500 base pairs up and downstream from the mutations were commercially synthesized. Each fragment also has additional secondary silent mutations to introduce novel MfeI and PstI restriction sites near the E1990A and H3369A mutations, respectively. Sequences are listed in Table S4. Fragments were cloned into pDS132 digested with SphI (New England Biolabs, \#R3182S) using Gibson Assembly (New England Biolabs, \#E2611S) according to manufacturer's instructions. Plasmids were recovered and propagated in DH5a $\lambda$ pir on LB supplemented with chloramphenicol and confirmed by sequencing.

\section{Transfer of E1990A, H2782A, and H3369A mutations to the V. cholerae chromosome}

The ACD E1990A and ABH H3369A mutations on the above pDS132-based plasmids were transferred to SM10 $\lambda$ pir. These mutations or the RID H2782A mutation from pSA129 were transferred to the $V$. cholerae chromosome by conjugation followed by $s a c B$-dependent counterselection for double homologous recombination as previously described $(2,60)$. To confirm recombinants gained the desired mutations, regions corresponding to the mutations were amplified by PCR, digested with the introduced novel restriction site, and products were separated on agarose gel. Presence of introduced mutations were also confirmed by sequencing.

\section{IL-8 enzyme-linked immunosorbent assay (ELISA)}

1- to $2 \times 10^{5}$ T84 cells seeded in a 12-well tissue culture treated plate were treated as described above for $2 \mathrm{hr}$. Media from inoculated cells was removed, cells were washed once with warm PBS and media changed to DMEM12 GlutaMAX media supplemented with serum, pen-strep, and $100 \mu \mathrm{g} \mathrm{mL}^{-1}$ gentamicin. Cells were incubated for an additional $20 \mathrm{hr}$ at $37^{\circ} \mathrm{C}$ in the presence of $5 \% \mathrm{CO}_{2}$. For IL-8 chemical inhibitor studies, $10 \mu \mathrm{M}$ of MAPK inhibitor or $0.33 \%$ DMSO control were added to T84 cells one hour prior to bacterial challenge. Inhibitors were reapplied following $2 \mathrm{hr}$ bacterial challenge. Media from the T84 cells was harvested and spun down at 20,000x $g$ for $1 \mathrm{~min}$ at $4^{\circ} \mathrm{C}$ and supernatant was collected. Concentration of IL-8 in cell media was measured using the IL-8 Human Matched Antibody Pair ELISA kit (ThermoFisher, \#CHC1303) following manufacturer's instructions.

\section{Western blot analysis of actin cross-linking and cell signaling pathways}

$1 \times 10^{6} \mathrm{~T} 84$ cells in a 6-well tissue culture dish or $1 \times 10^{5} \mathrm{HeLa}$ cells in a 12-well tissue culture dish were treated with various $V$. cholerae strains as described above for $2 \mathrm{hr}$. Cells were washed once with cold PBS and removed from the plate in $150 \mu \mathrm{L}$ lysis buffer (150 $\mathrm{mM} \mathrm{NaCl}, 20 \mathrm{mM}$ TRIS pH 7.5, 1\% Triton X-100, and Pierce Protease and Phosphatase inhibitor added prior to use) using a cell scrapper. Lysates were incubated on ice for $15 \mathrm{~min}$ and then clarified by centrifugation at $20,000 \mathrm{xg}$ at $4{ }^{\circ} \mathrm{C}$ for $10 \mathrm{~min}$. Concentration of protein 
in the collected supernatant fluid determined using the bicinchoninic (BCA) assay (ThermoFisher, \#23227). Specific samples were also collected directly in $150 \mu \mathrm{L} 2 \mathrm{X}$ SDS loading buffer and normalized by volume where indicated. Normalized samples were boiled for $5 \mathrm{~min}$ at $95^{\circ} \mathrm{C}$ in SDS loading buffer and protein separated on either a $10 \%$ or $15 \%$ SDSpolyacrylamide gel. Proteins were transferred to nitrocellulose membranes and blocked in TBS (10 mM Tris $\mathrm{pH}=7.4,150 \mathrm{mM} \mathrm{NaCl})$ with $5 \%(\mathrm{w} / \mathrm{v})$ milk for 1 hour. Membranes were washed with TBS and then incubated in indicated primary antibodies 1:1000 in TBS with $5 \%$ (w/v) bovine serum album (BSA, Fisher Bioreagents) overnight at $4{ }^{\circ} \mathrm{C}$. Membranes were washed with TBS and probed in 1:10,000 IRDye 800CW/680LT secondary antibody for 1 hour before being washed again and imaged using the LI-COR Bioscience Odyssey imaging system. Quantification of band density was conducted using Fiji/ImageJ.

\section{Rac1 G-LISA}

$1 \times 10^{6} \mathrm{~T} 84$ cells in a 6-well tissue culture dish were treated with various $V$. cholerae strains as described above for $2 \mathrm{hr}$. Cells were washed once with cold PBS and resuspended in 100 $\mu \mathrm{L}$ G-LISA lysis buffer. Activation of Rac1 was quantified from equal concentration of lysate using the Rac1 G-LISA Activation Assay (Cytoskeleton, \#BK128) following manufacturers protocol. Percent change in active Rac1 compared to $\Delta r t x$ strain was

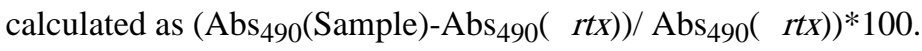

\section{RNA-seq}

The stranded mRNA-seq was conducted in the Northwestern University NUSeq Core Facility. Briefly, total RNA examples were checked for quality using RINs generated from Agilent Bioanalyzer 2100. RNA quantity was determined with Qubit fluorometer. The Illumina TruSeq Stranded mRNA Library Preparation Kit was used to prepare sequencing libraries from $750 \mathrm{ng}$ of high-quality RNA samples $(\mathrm{RIN}=10)$. The Kit procedure was performed without modifications. This procedure includes mRNA purification and fragmentation, cDNA synthesis, 3' end adenylation, Illumina adapter ligation, library PCR amplification and validation. 1llumina NextSeq 500 Sequencer was used to sequence the libraries with the production of single-end, 75 bp reads.

The quality of DNA reads, in fastq format, was evaluated using FastQC. Adapters were trimmed, and reads of poor quality or aligning to rRNA sequences were filtered. The cleaned reads were aligned to the human reference genome using STAR (61). Read counts for each gene were calculated using htseq-count (62). Normalization and differential expression were determined using DESeq2 (63). The cutoff for determining significantly differentially expressed genes was an FDR-adjusted p-value less than 0.05. A pathway analysis was performed on both gene lists using GeneCoDis (64-66) to identify pathways that are enriched with genes that are upregulated and downregulated.

\section{Quantitative RT-PCR}

$1 \times 10^{6} \mathrm{~T} 84$ cells in a 6-well tissue culture treated plate were treated with $V$. cholerae as described above. At indicated time points, mRNA was harvested using the Qiagen RNeasy kit (Qiagen, \#74014) following manufacturer's instructions. RNA isolated was measured using a Nano-drop 100 spectrophotometer. Reverse transcription was performed using 
random hexamers (Roche) and Superscript III Reverse Transcriptase (Invitrogen, \#18080044) in the presence of RNase OUT (Invitrogen, \#10777019) or RNasin (Promega, \#N2611) under the following conditions: $25^{\circ} \mathrm{C}$ for $5 \mathrm{~min}$, then $55^{\circ} \mathrm{C}$ for $60 \mathrm{~min}, 95^{\circ} \mathrm{C}$ for 5 min. Remaining RNA was hydrolyzed using $1 \mathrm{~N} \mathrm{NaOH}$. Quantitative PCR was performed using iQ SYBR Green supermix (Bio-Rad, \#1708880) on the iQ5 Multicolor RealTime PCR Detection System using gene specific primers indicated in Table S5. Relative change in gene expression compared to PBS control was determined the $\Delta \Delta \mathrm{CT}$ method (67).

\section{Quantification and statistical analysis}

All experiments were done at least in triplicate, and quantitative results are reported as the mean \pm standard deviation (s.d.). Statistical analysis was conducted assuming normal distribution using GraphPad Prism v6.0 as detailed in the figure legends. Statistical differences in ELISA and qPCR results were determined by one-way ANOVA followed by Tukey's multiple comparison's test. Statistical difference in results comparing suppression of ERK, p38, and JNK signaling by various $V$. cholerae strains compared to the ACD Active only strain were determined using Student's t-tests.

\section{Supplementary Material}

Refer to Web version on PubMed Central for supplementary material.

\section{Acknowledgements}

We would like to thank the Northwestern University Center for Genetic Medicine NUSeq core facility, especially Xinkun Wang and Matthew Schipma, for technical assistance, bioinformatic analysis of the RNA-sequencing experiments and DNA sequencing. Dr. Sheng Wang is acknowledged for statistical review. We would like to thank members of the Satchell lab for their valuable input and technical support and Dr. Nicholas Cianciotto and Dr. Gail Hecht for review of the manuscript.

Funding: This work was supported by the NIH Ruth L. Kirschstein Institutional National Research Service Award Training Grant in Immunology and Microbial Pathogenesis T32AI007476 (to P.J.W.) and NIH grants R01AI092825 and R01AI098369 (to K.J.F.S.).

\section{REFERENCES AND NOTES}

1. Satchell KJ, Multifunctional-autoprocessing repeats-in-toxin (MARTX) Toxins of Vibrios. Microbiol Spectr 3, (2015).

2. Dolores JS, Agarwal S, Egerer M, Satchell KJ, Vibrio cholerae MARTX toxin heterologous translocation of beta-lactamase and roles of individual effector domains on cytoskeleton dynamics. Mol Microbiol 95, 590-604 (2015). [PubMed: 25427654]

3. Kim BS, Gavin HE, Satchell KJ, Distinct roles of the repeat-containing regions and effector domains of the Vibrio vulnificus multifunctional-autoprocessing repeats-in-toxin (MARTX) toxin. MBio 6, (2015).

4. Kim YR, Lee SE, Kook H, Yeom JA, Na HS, Kim SY, Chung SS, Choy HE, Rhee JH, Vibrio vulnificus RTX toxin kills host cells only after contact of the bacteria with host cells. Cell Microbiol 10, 848-862 (2008). [PubMed: 18005241]

5. Egerer M, Satchell KJ, Inositol hexakisphosphate-induced autoprocessing of large bacterial protein toxins. PLoS Pathog 6, e1000942 (2010). [PubMed: 20628577]

6. Prochazkova K, Shuvalova LA, Minasov G, Voburka Z, Anderson WF, Satchell KJ, Structural and molecular mechanism for autoprocessing of MARTX toxin of Vibrio cholerae at multiple sites. J Biol Chem 284, 26557-26568 (2009). [PubMed: 19620709] 
7. Shen A, Lupardus PJ, Albrow VE, Guzzetta A, Powers JC, Garcia KC, Bogyo M, Mechanistic and structural insights into the proteolytic activation of Vibrio cholerae MARTX toxin. Nat Chem Biol 5, 469-478 (2009). [PubMed: 19465933]

8. Kim BS, The Modes of Action of MARTX Toxin Effector Domains. Toxins (Basel) 10, (2018).

9. Woida PJ, Satchell KJF, Coordinated delivery and function of bacterial MARTX toxin effectors. Mol Microbiol 107, 133-141 (2018). [PubMed: 29114985]

10. Ali M, Lopez AL, You YA, Kim YE, Sah B, Maskery B, Clemens J, The global burden of cholera. Bull World Health Organ 90, 209-218A (2012). [PubMed: 22461716]

11. Olivier V, Queen J, Satchell KJ, Successful small intestine colonization of adult mice by Vibrio cholerae requires ketamine anesthesia and accessory toxins. PLoS One 4, e7352 (2009). [PubMed: 19812690]

12. Olivier V, Haines GK 3rd, Tan Y, Satchell KJ, Hemolysin and the multifunctional autoprocessing RTX toxin are virulence factors during intestinal infection of mice with Vibrio cholerae El Tor O1 strains. Infect Immun 75, 5035-5042 (2007). [PubMed: 17698573]

13. Weill FX, Domman D, Njamkepo E, Tarr C, Rauzier J, Fawal N, Keddy KH, Salje H, Moore S, Mukhopadhyay AK, Bercion R, Luquero FJ, Ngandjio A, Dosso M, Monakhova E, Garin B, Bouchier C, Pazzani C, Mutreja A, Grunow R, Sidikou F, Bonte L, Breurec S, Damian M, Njanpop-Lafourcade BM, Sapriel G, Page AL, Hamze M, Henkens M, Chowdhury G, Mengel M, Koeck JL, Fournier JM, Dougan G, Grimont PAD, Parkhill J, Holt KE, Piarroux R, Ramamurthy T, Quilici ML, Thomson NR, Genomic history of the seventh pandemic of cholera in Africa. Science 358, 785-789 (2017). [PubMed: 29123067]

14. Queen J, Satchell KJ, Neutrophils are essential for containment of Vibrio cholerae to the intestine during the proinflammatory phase of infection. Infect Immun 80, 2905-2913 (2012). [PubMed: 22615254]

15. Dolores J, Satchell KJ, Analysis of Vibrio cholerae genome sequences reveals unique $r t x A$ variants in environmental strains and an $r t x A$-null mutation in recent altered El Tor isolates. MBio 4, e00624 (2013). [PubMed: 23592265]

16. Fullner KJ, Mekalanos JJ, In vivo covalent cross-linking of cellular actin by the Vibrio cholerae RTX toxin. EMBO J 19, 5315-5323 (2000). [PubMed: 11032799]

17. Sheahan KL, Cordero CL, Satchell KJ, Identification of a domain within the multifunctional Vibrio cholerae RTX toxin that covalently cross-links actin. Proc Natl Acad Sci U S A 101, 9798-9803 (2004). [PubMed: 15199181]

18. Kudryashov DS, Cordero CL, Reisler E, Satchell KJ, Characterization of the enzymatic activity of the actin cross-linking domain from the Vibrio cholerae MARTX $V c$ toxin. J Biol Chem 283, 445452 (2008). [PubMed: 17951576]

19. Heisler DB, Kudryashova E, Grinevich DO, Suarez C, Winkelman JD, Birukov KG, Kotha SR, Parinandi NL, Vavylonis D, Kovar DR, Kudryashov DS, ACD toxin-produced actin oligomers poison formin-controlled actin polymerization. Science 349, 535-539 (2015). [PubMed: 26228148]

20. Zhou Y, Huang C, Yin L, Wan M, Wang X, Li L, Liu Y, Wang Z, Fu P, Zhang N, Chen S, Liu X, Shao F, Zhu Y, N(epsilon)-Fatty acylation of Rho GTPases by a MARTX toxin effector. Science 358, 528-531 (2017). [PubMed: 29074776]

21. Sheahan KL, Satchell KJ, Inactivation of small Rho GTPases by the multifunctional RTX toxin from Vibrio cholerae. Cell Microbiol 9, 1324-1335 (2007). [PubMed: 17474905]

22. Agarwal S, Kim H, Chan RB, Agarwal S, Williamson R, Cho W, Paolo GD, Satchell KJ, Autophagy and endosomal trafficking inhibition by Vibrio cholerae MARTX toxin phosphatidylinositol-3-phosphate-specific phospholipase A1 activity. Nat Commun 6, 8745 (2015). [PubMed: 26498860]

23. Kagnoff MF, Eckmann L, Epithelial cells as sensors for microbial infection. J Clin Invest 100, 610 (1997). [PubMed: 9202050]

24. Peterson LW, Artis D, Intestinal epithelial cells: regulators of barrier function and immune homeostasis. Nat Rev Immunol 14, 141-153 (2014). [PubMed: 24566914] 
25. Qadri F, Raqib R, Ahmed F, Rahman T, Wenneras C, Das SK, Alam NH, Mathan MM, Svennerholm AM, Increased levels of inflammatory mediators in children and adults infected with Vibrio cholerae O1 and O139. Clin Diagn Lab Immunol 9, 221-229 (2002). [PubMed: 11874856]

26. Bishop AL, Patimalla B, Camilli A, Vibrio cholerae-induced inflammation in the neonatal mouse cholera model. Infect Immun 82, 2434-2447 (2014). [PubMed: 24686062]

27. Zhou X, Gao DQ, Michalski J, Benitez JA, Kaper JB, Induction of interleukin-8 in T84 cells by Vibrio cholerae. Infect Immun 72, 389-397 (2004). [PubMed: 14688120]

28. Rodriguez BL, Rojas A, Campos J, Ledon T, Valle E, Toledo W, Fando R, Differential interleukin-8 response of intestinal epithelial cell line to reactogenic and nonreactogenic candidate vaccine strains of Vibrio cholerae. Infect Immun 69, 613-616 (2001). [PubMed: 11119564]

29. Harrison LM, Rallabhandi P, Michalski J, Zhou X, Steyert SR, Vogel SN, Kaper JB, Vibrio cholerae flagellins induce Toll-like receptor 5-mediated interleukin-8 production through mitogenactivated protein kinase and NF-kappaB activation. Infect Immun 76, 5524-5534 (2008). [PubMed: 18809662]

30. Soriani M, Bailey L, Hirst TR, Contribution of the ADP-ribosylating and receptor-binding properties of cholera-like enterotoxins in modulating cytokine secretion by human intestinal epithelial cells. Microbiology 148, 667-676 (2002). [PubMed: 11882700]

31. Bobo LD, El Feghaly RE, Chen YS, Dubberke ER, Han Z, Baker AH, Li J, Burnham CA, Haslam DB, MAPK-activated protein kinase 2 contributes to Clostridium difficile-associated inflammation. Infect Immun 81, 713-722 (2013). [PubMed: 23264053]

32. Fullner KJ, Boucher JC, Hanes MA, Haines GK 3rd, Meehan BM, Walchle C, Sansonetti PJ, Mekalanos JJ, The contribution of accessory toxins of Vibrio cholerae O1 El Tor to the proinflammatory response in a murine pulmonary cholera model. J Exp Med 195, 1455-1462 (2002). [PubMed: 12045243]

33. Satchell KJ, Jones CJ, Wong J, Queen J, Agarwal S, Yildiz FH, Phenotypic Analysis Reveals that the 2010 Haiti Cholera Epidemic Is Linked to a Hypervirulent Strain. Infect Immun 84, 2473 2481 (2016). [PubMed: 27297393]

34. Schwartz M, Rho signalling at a glance. J Cell Sci 117, 5457-5458 (2004). [PubMed: 15509861]

35. Woolery AR, Yu X, LaBaer J, Orth K, AMPylation of Rho GTPases subverts multiple host signaling processes. J Biol Chem 289, 32977-32988 (2014). [PubMed: 25301945]

36. Kudryashova E, Heisler DB, Williams B, Harker AJ, Shafer K, Quinlan ME, Kovar DR, Vavylonis D, Kudryashov DS, Actin Cross-Linking Toxin Is a Universal Inhibitor of Tandem-Organized and Oligomeric G-Actin Binding Proteins. Curr Biol 28, 1536-1547 e1539 (2018). [PubMed: 29731300]

37. Cordero CL, Kudryashov DS, Reisler E, Satchell KJ, The Actin cross-linking domain of the Vibrio cholerae RTX toxin directly catalyzes the covalent cross-linking of actin. J Biol Chem 281, 32366-32374 (2006). [PubMed: 16954226]

38. Gavin HE, Satchell KJ, MARTX toxins as effector delivery platforms. Pathog Dis 73, ftv092 (2015). [PubMed: 26472741]

39. Boardman BK, Meehan BM, Fullner Satchell KJ, Growth phase regulation of Vibrio cholerae RTX toxin export. J Bacteriol 189, 1827-1835 (2007). [PubMed: 17189368]

40. Alam M, Islam A, Bhuiyan NA, Rahim N, Hossain A, Khan GY, Ahmed D, Watanabe H, Izumiya H, Faruque AS, Akanda AS, Islam S, Sack RB, Huq A, Colwell RR, Cravioto A, Clonal transmission, dual peak, and off-season cholera in Bangladesh. Infect Ecol Epidemiol 1, (2011).

41. Son MS, Megli CJ, Kovacikova G, Qadri F, Taylor RK, Characterization of Vibrio cholerae O1 El Tor biotype variant clinical isolates from Bangladesh and Haiti, including a molecular genetic analysis of virulence genes. J Clin Microbiol 49, 3739-3749 (2011). [PubMed: 21880975]

42. Russell R, Wang H, Benitez JA, Silva AJ, Deletion of gene encoding the nucleoid-associated protein H-NS unmasks hidden regulatory connections in El Tor biotype Vibrio cholerae. Microbiology 164, 998-1003 (2018). [PubMed: 29813015]

43. Gavin HE, Beubier NT, Satchell KJ, The Effector Domain Region of the Vibrio vulnificus MARTX Toxin Confers Biphasic Epithelial Barrier Disruption and Is Essential for Systemic Spread from the Intestine. PLoS Pathog 13, e1006119 (2017). [PubMed: 28060924] 
44. Chen CL, Chien SC, Leu TH, Harn HI, Tang MJ, Hor LI, Vibrio vulnificus MARTX cytotoxin causes inactivation of phagocytosis-related signaling molecules in macrophages. J Biomed Sci 24, 58 (2017). [PubMed: 28822352]

45. Mahida YR, Makh S, Hyde S, Gray T, Borriello SP, Effect of Clostridium difficile toxin A on human intestinal epithelial cells: induction of interleukin 8 production and apoptosis after cell detachment. Gut 38, 337-347 (1996). [PubMed: 8675084]

46. Xu H, Yang J, Gao W, Li L, Li P, Zhang L, Gong YN, Peng X, Xi JJ, Chen S, Wang F, Shao F, Innate immune sensing of bacterial modifications of Rho GTPases by the Pyrin inflammasome. Nature 513, 237-241 (2014). [PubMed: 24919149]

47. Marat AL, Haucke V, Phosphatidylinositol 3-phosphates-at the interface between cell signalling and membrane traffic. EMBO J 35, 561-579 (2016). [PubMed: 26888746]

48. Baumeister MA, Martinu L, Rossman KL, Sondek J, Lemmon MA, Chou MM, Loss of phosphatidylinositol 3-phosphate binding by the C-terminal Tiam-1 pleckstrin homology domain prevents in vivo Rac1 activation without affecting membrane targeting. J Biol Chem 278, 1145711464 (2003). [PubMed: 12525493]

49. Remorino A, De Beco S, Cayrac F, Di Federico F, Cornilleau G, Gautreau A, Parrini MC, Masson JB, Dahan M, Coppey M, Gradients of Rac1 Nanoclusters Support Spatial Patterns of Rac1 Signaling. Cell Rep 21, 1922-1935 (2017). [PubMed: 29141223]

50. Campa CC, Ciraolo E, Ghigo A, Germena G, Hirsch E, Crossroads of PI3K and Rac pathways. Small GTPases 6, 71-80 (2015). [PubMed: 25942647]

51. Fehrenbacher N, Bar-Sagi D, Philips M, Ras/MAPK signaling from endomembranes. Mol Oncol 3, 297-307 (2009). [PubMed: 19615955]

52. Martinez-Lopez N, Athonvarangkul D, Mishall P, Sahu S, Singh R, Autophagy proteins regulate ERK phosphorylation. Nat Commun 4, 2799 (2013). [PubMed: 24240988]

53. Ma AT, Mekalanos JJ, In vivo actin cross-linking induced by Vibrio cholerae type VI secretion system is associated with intestinal inflammation. Proc Natl Acad Sci U S A 107, 4365-4370 (2010). [PubMed: 20150509]

54. Brooks AB, Humphreys D, Singh V, Davidson AC, Arden SD, Buss F, Koronakis V, MYO6 is targeted by Salmonella virulence effectors to trigger PI3-kinase signaling and pathogen invasion into host cells. Proc Natl Acad Sci U S A 114, 3915-3920 (2017). [PubMed: 28348208]

55. Cain RJ, Hayward RD, Koronakis V, Deciphering interplay between Salmonella invasion effectors. PLoS Pathog 4, e1000037 (2008). [PubMed: 18389058]

56. Chung LK, Park YH, Zheng Y, Brodsky IE, Hearing P, Kastner DL, Chae JJ, Bliska JB, The Yersinia Virulence Factor YopM Hijacks Host Kinases to Inhibit Type III Effector-Triggered Activation of the Pyrin Inflammasome. Cell Host Microbe 20, 296-306 (2016). [PubMed: 27569559]

57. Kwak JS, Jeong HG, Satchell KJ, Vibrio vulnificus rtxA1 gene recombination generates toxin variants with altered potency during intestinal infection. Proc Natl Acad Sci U S A 108, 16451650 (2011). [PubMed: 21220343]

58. Murciano C, Lee CT, Fernandez-Bravo A, Hsieh TH, Fouz B, Hor LI, Amaro C, MARTX Toxin in the Zoonotic Serovar of Vibrio vulnificus Triggers an Early Cytokine Storm in Mice. Front Cell Infect Microbiol 7, 332 (2017). [PubMed: 28775962]

59. Gavin HE, Satchell KJF, RRSP and RID Effector Domains Dominate Virulence Impact of Vibrio vulnificus MARTX Toxin. J Infect Dis, (2018).

60. Ahrens S, Geissler B, Satchell KJ, Identification of a His-Asp-Cys catalytic triad essential for function of the Rho inactivation domain (RID) of Vibrio cholerae MARTX toxin. J Biol Chem 288, 1397-1408 (2013). [PubMed: 23184949]

61. Dobin A, Davis CA, Schlesinger F, Drenkow J, Zaleski C, Jha S, Batut P, Chaisson M, Gingeras TR, STAR: ultrafast universal RNA-seq aligner. Bioinformatics 29, 15-21 (2013). [PubMed: 23104886]

62. Anders S, Pyl PT, Huber W, HTSeq--a Python framework to work with high-throughput sequencing data. Bioinformatics 31, 166-169 (2015). [PubMed: 25260700]

63. Love MI, Huber W, Anders S, Moderated estimation of fold change and dispersion for RNA-seq data with DESeq2. Genome Biol 15, 550 (2014). [PubMed: 25516281] 
64. Tabas-Madrid D, Nogales-Cadenas R, Pascual-Montano A, GeneCodis3: a non-redundant and modular enrichment analysis tool for functional genomics. Nucleic Acids Res 40, W478-483 (2012). [PubMed: 22573175]

65. Nogales-Cadenas R, Carmona-Saez P, Vazquez M, Vicente C, Yang X, Tirado F, Carazo JM, Pascual-Montano A, GeneCodis: interpreting gene lists through enrichment analysis and integration of diverse biological information. Nucleic Acids Res 37, W317-322 (2009). [PubMed: 19465387]

66. Carmona-Saez P, Chagoyen M, Tirado F, Carazo JM, Pascual-Montano A, GENECODIS: a webbased tool for finding significant concurrent annotations in gene lists. Genome Biol 8, R3 (2007). [PubMed: 17204154]

67. Livak KJ, Schmittgen TD, Analysis of relative gene expression data using real-time quantitative PCR and the 2(-Delta Delta C(T)) Method. Methods 25, 402-408 (2001). [PubMed: 11846609] 

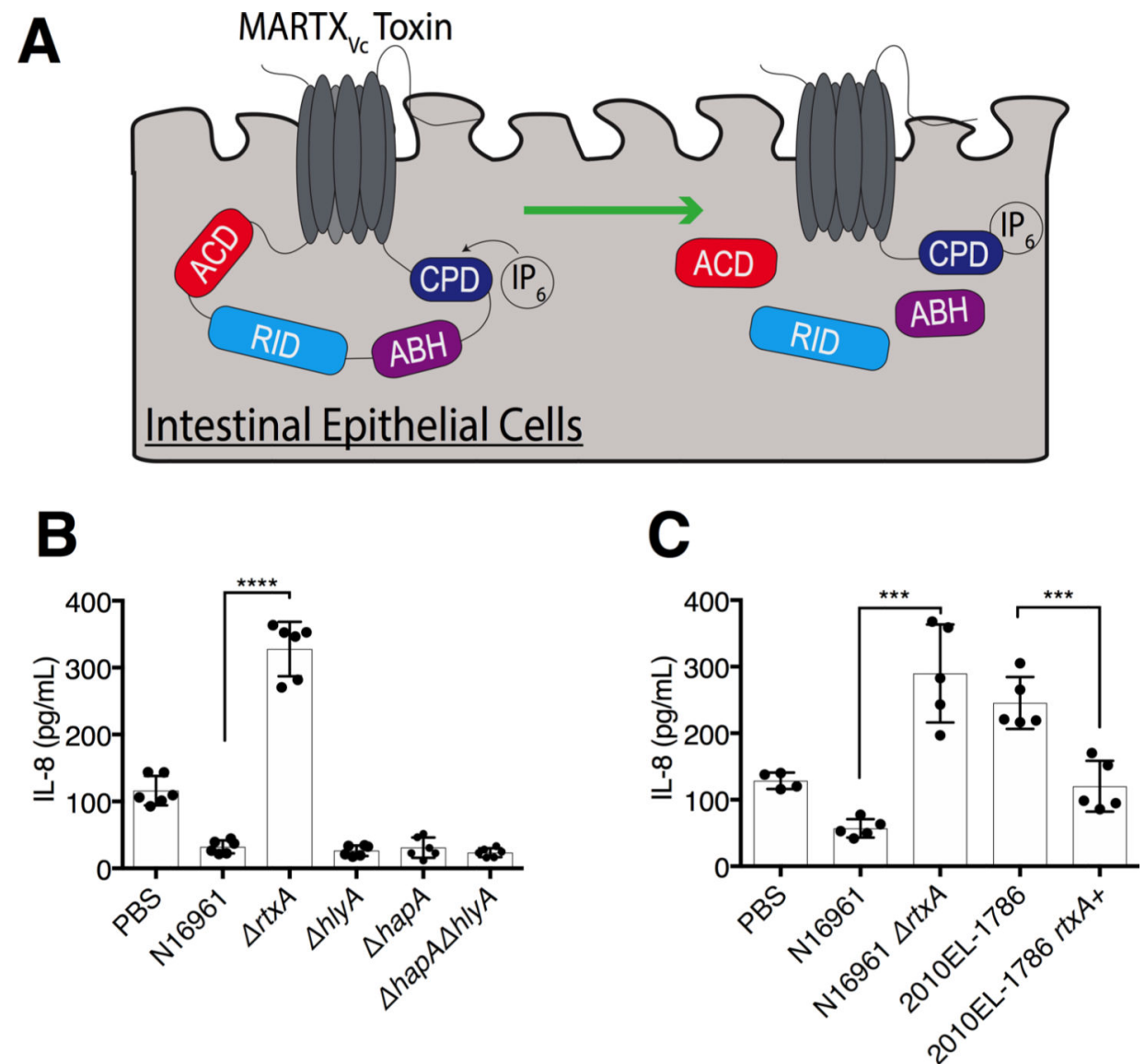

Fig. 1. The MARTX ${ }_{V c}$ toxin, but not other $V$. cholerae accessory toxins, suppresses IL-8 secretion in IECs.

(A) Schematic of MARTX $V_{c}$ toxin effector domains in IECs. The N- and C-terminal regions of the MARTX $_{V c}$ toxin form a pore in the target eukaryotic cell membrane through which the central effector domains (ACD, RID, and $\mathrm{ABH}$ ) and the protease CPD translocate into the host cell. $\mathrm{CPD}$ binds to InsP $_{6}$, which activates the domain's autoproteolytic activity to separate and release the three effector domains from the holotoxin. (B, C) Quantification of IL-8 secretion into the medium by T84 human IECs inoculated with $V$. cholerae strain N16961 or the indicated N16961 variants (B) or with the 2010EL-1786 clinical isolate, which contains a natural premature stop codon in MARTX $_{V c}$, and a variant of this strain lacking the premature stop codon (C). Data in (B) and (C) are pooled data from $n=3$ independent experiments reported as means \pm standard deviation (s.d.). Statistical significance of indicated sample pairs determined using Student's $t$-test $(* * * \mathrm{p}<0.001$, $* * * * \mathrm{p}<0.0001)$. 
A

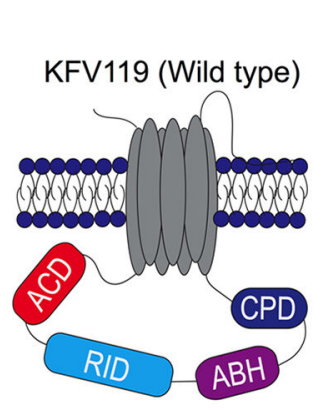

B

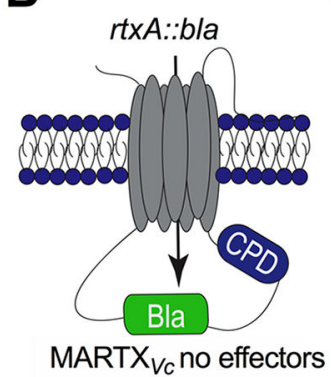

C

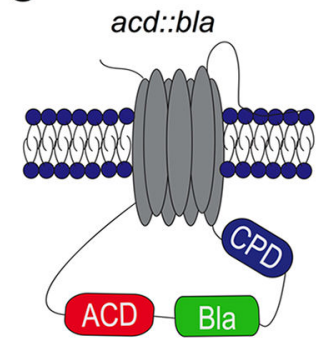

D

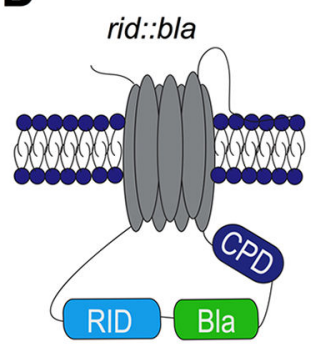

E

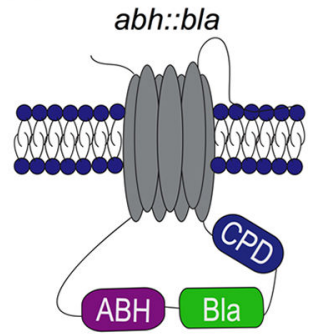

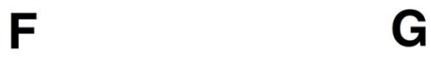

G
H

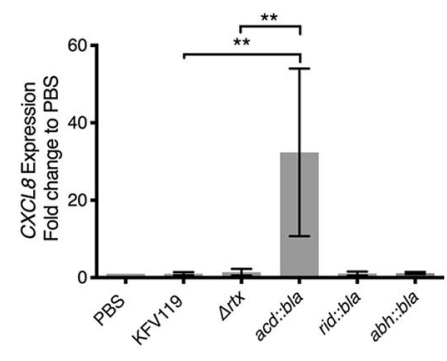

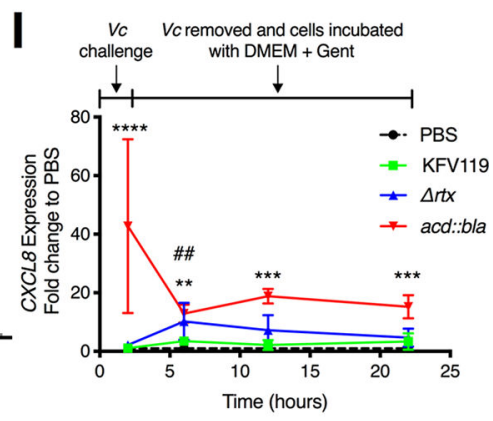
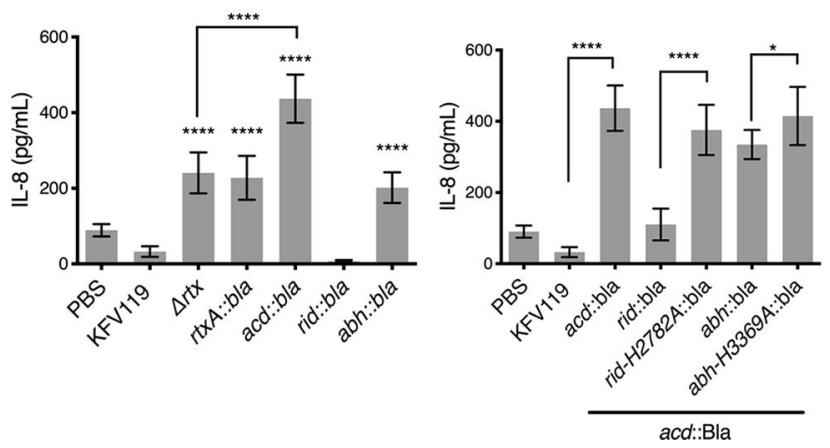

Fig. 2. The MARTX ${ }_{V c}$ toxin effector domains, and not the pore, differentially regulate IL-8 secretion in IECs.

(A-E) Schematics of MARTX $_{V c}$ toxin effector arrangements in wild-type $V$. cholerae (A) and in strains modified to translocate $\beta$-lactamase (Bla) in place of all three effector domains (B) and in single effector gain-of-function strains (2) in which each effector domain was individually restored (C-E). (F, G) Quantification of IL-8 secretion from T84 IECs inoculated with one (F) or two (G) $V$. cholerae effector-free Bla or single effector gain-offunction strains as indicated. (H) Quantification of $C X C L 8$ expression by qPCR in T84 cells at 120 min post-inoculation with the indicated $V$. cholerae strains. (I) Quantification of $C X C L 8$ expression by qPCR with bacteria removal at $2 \mathrm{hr}$ and then assayed at the indicated times from antibiotic-containing media. Data for all panels are pooled from $n=3$ independent experiments reported as means \pm s.d. Statistical significance determined by One-way ANOVA with Tukey's multiple comparison's test for samples as indicated $(* \mathrm{p}<0.5, * * \mathrm{p}<0.01, * * * \mathrm{p}<0.001, * * * * \mathrm{p}<0.0001)$. In (I), PBS mock-treated sample was normalized to 1, asterisks indicate multiple comparisons between acd::bla and PBS, and \#\# $(\mathrm{p}<0.001)$ for multiple comparison between $\Delta r t x$ and PBS set at the same time point. 

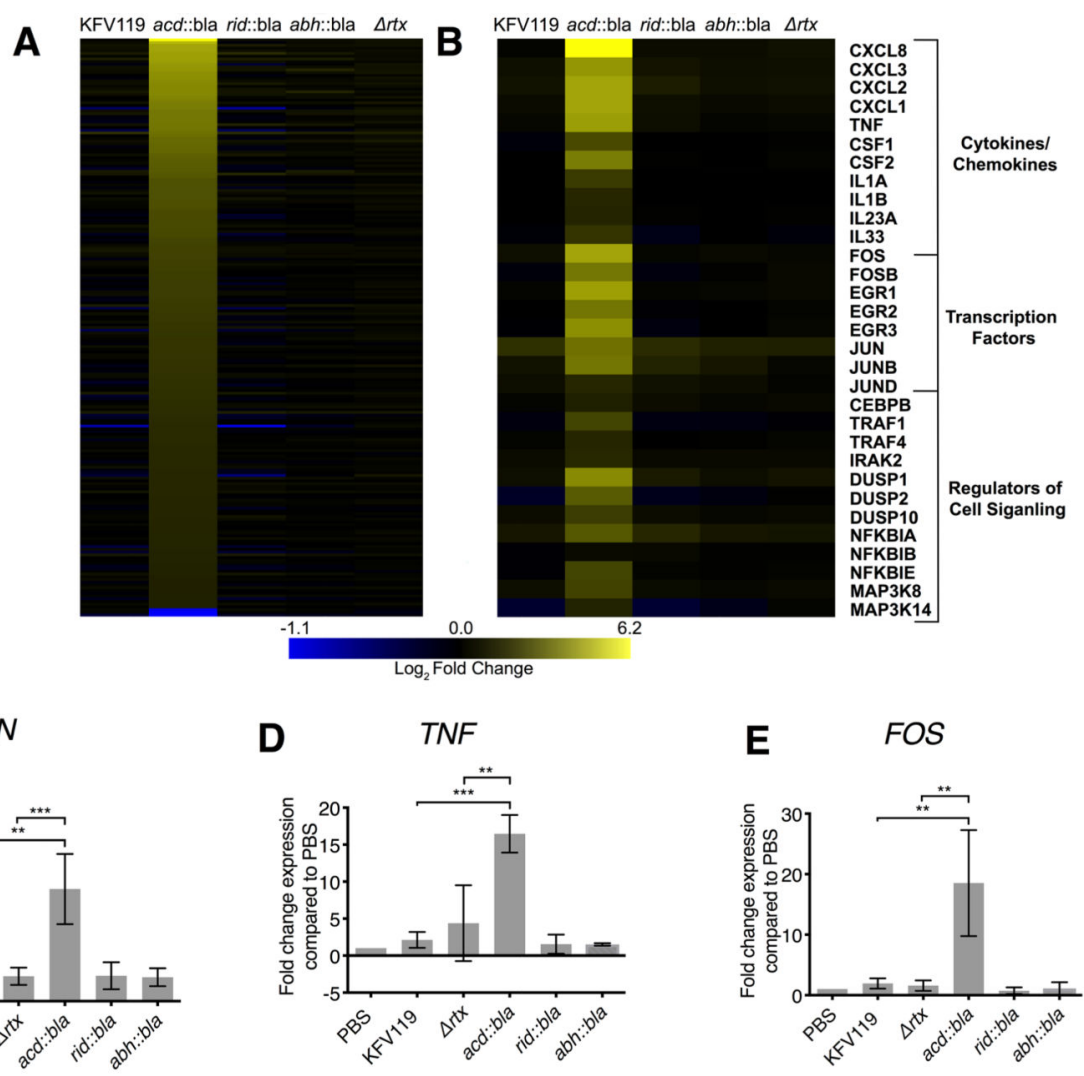

\begin{tabular}{l|l} 
TNF & \\
CSF1 & Cytokines/
\end{tabular}

Chemokines

IL1A

IL1B

IL 233

FOS

FOSB
EGR1

EGR1

\begin{tabular}{l|l} 
EGR2 & Transcriptio \\
EGR3
\end{tabular}

EGR3

JUNB

Factors

CEBPB

RAF1

TRAF4
IRAK2

DUSP1

DUSP2 Regulators of

DUSP10 Cell Siganling

NFKBIA

NFKBIB

MAP3K8

MAP3K8

C JUN

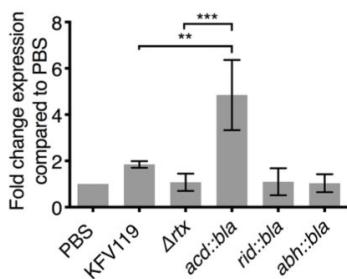

F $\quad \mathrm{CXCL3}$

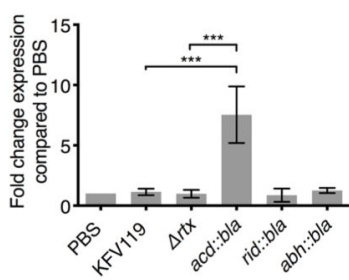

G

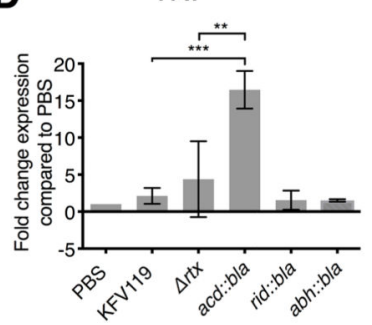

E FOS

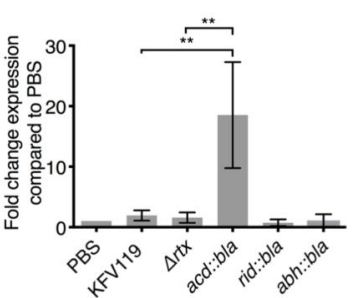

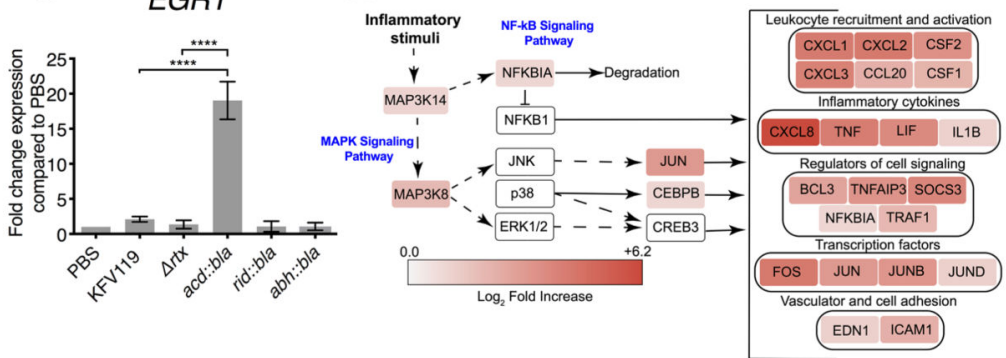

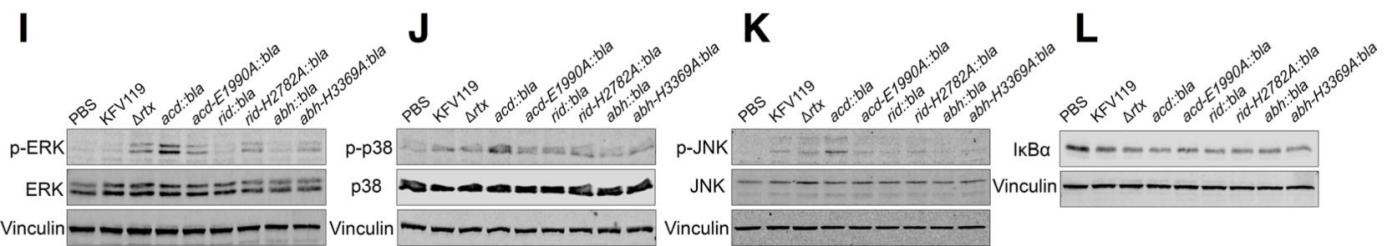

Fig. 3. ACD induces a global proinflammatory response that is silenced by co-delivery with RID and $\mathrm{ABH}$.

(A) Heat map of differentially expressed genes identified in RNA-sequencing analysis of T84 cells inoculated with the indicated $V$. cholerae strains. (B) Heat map of differentially expressed inflammatory genes identified from RNA-seq analysis. $n=2$ independent experiments.

Heat maps represent statistically significant differentially expressed genes with a $-1 \leq 0 \leq 1$ $\log _{2}$ fold change cut off. (C-G) qPCR validation of select differentially expressed genes 
from (A) and (B), $n=3$ independent experiments. Data are reported as the mean \pm s.d. (** $\mathrm{p}<0.01, * * * \mathrm{p}<0.001, * * * * \mathrm{p}<0.0001$, One-way ANOVA with Tukey's multiple comparison's test for samples as indicated. No other strains induced a statistically different response compared to the normalized PBS control or another strain).

(H) ACD induced differential expression of genes involved in the regulation of or downstream of the MAPK and NF- $\mathrm{kB}$ signaling pathways as identified from RNA-seq analysis.

(I-L) Western blot analysis of phosphorylated ERK (p-ERK, I), phosphorylated p38 (p-p38, $\mathrm{J}$ ), phosphorylated JNK (p-JNK, K), and I $\kappa$ Ba degradation (L) in T84 cells inoculated with the indicated $V$. cholerae strains. Blots are representative of $n=3$ independent experiments with quantification shown in Fig. S2A-D. 


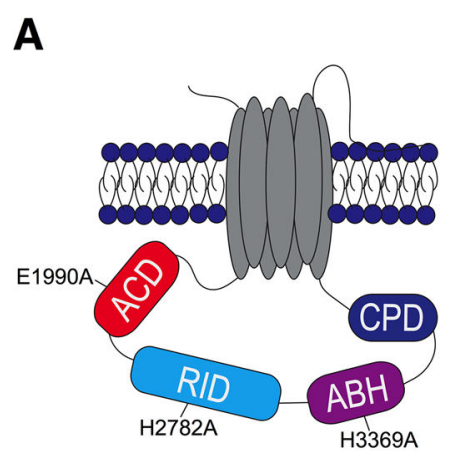

D

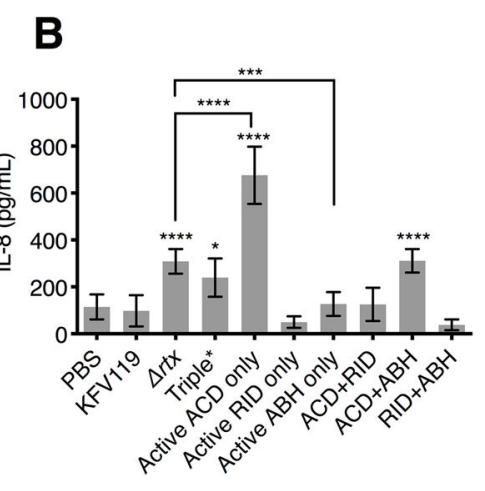

E

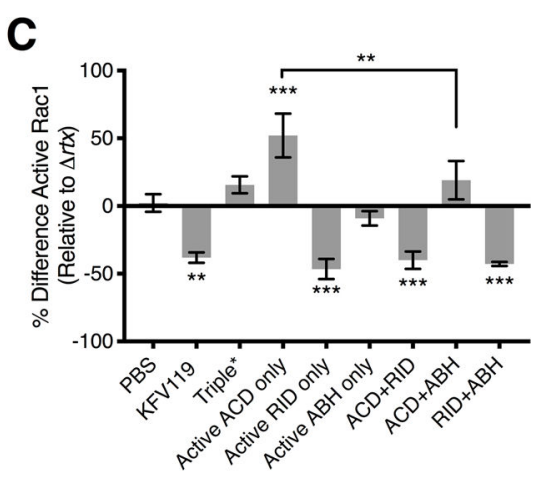

$\mathbf{F}$
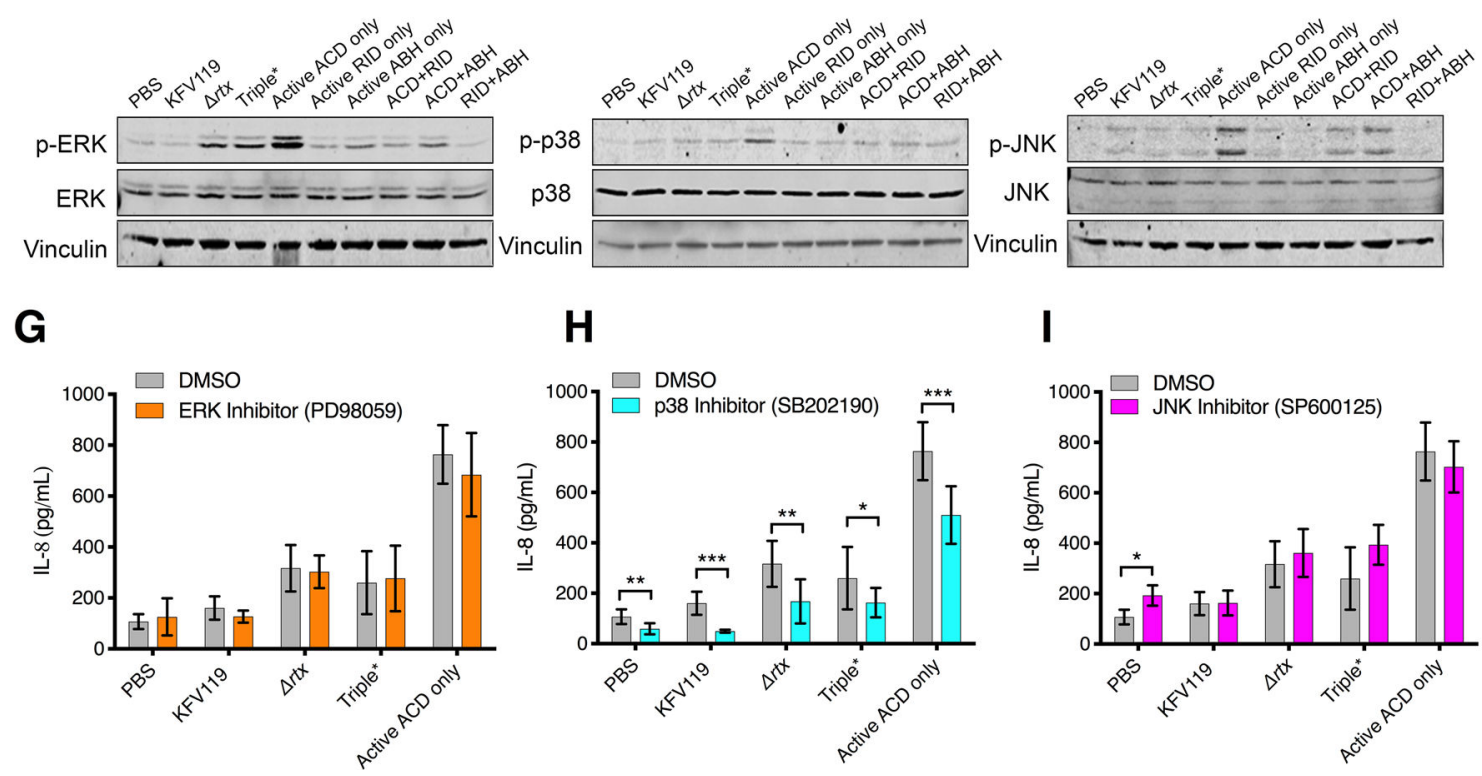

Fig. 4. Both RID and ABH suppress ACD-induced proinflammatory responses.

(A) Schematic of MARTX ${ }_{V c}$ toxin identifying catalytic residues mutated in single, double, and triple catalytically inactive MARTX $_{V_{c}}$ toxin effector strains. $(\mathbf{B}, \mathbf{C})$ Quantification of IL-8 secretion (B) or active (GTP-bound) Rac1 (C) measured from T84 cells inoculated with the indicated $V$. cholerae strains. In $(C)$, raw absorbances were normalized as the percent difference of active Rac1 relative to active Rac1 induced by the $\Delta r t x$ strain to identify effector -specific changes. Data from $n=3$ independent experiments reported as mean \pm s.d. $(* \mathrm{p}<0.05$, One-way ANOVA with Tukey's multiple comparison's test between cells treated with the indicated strain and PBS or for samples indicated, $(* * \mathrm{p}<0.01, * * * \mathrm{p}<0.001$, $* * * * \mathrm{p}<0.0001)$. (D-F) Western blot analysis of phosphorylated ERK(p-ERK, D), phosphorylated p38 (p-p38, E), and phosphorylated JNK (p-JNK, F) in T84 cells inoculated with the indicated $V$. cholerae strains. Blots are representative of $n=3$ independent experiments. Quantification of blot data is presented in Fig. S6. (G-I) Quantification of IL-8 secretion measured from T84 cells pre-treated with ERK inhibitor PD98059 (G), p38 inhibitor SB202190 (H), or JNK inhibitor SP600125 (I). Data pooled from $n=3$ independent experiments reported as means \pm s.d. $(* \mathrm{p}<0.05, * * \mathrm{p}<0.01, * * * * \mathrm{p}<0.0001$, Student's $t$-test for paired samples as indicated). 


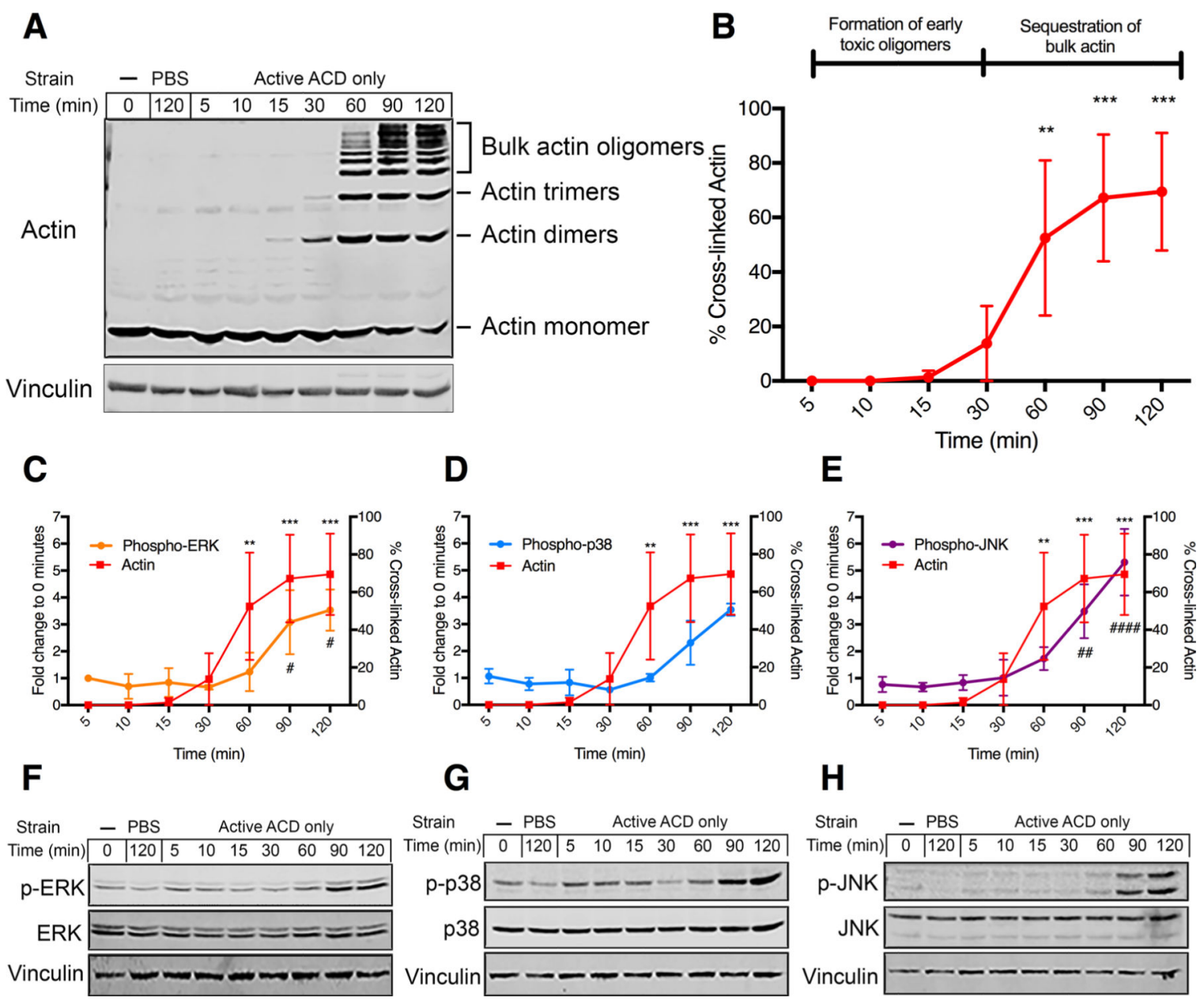

Fig. 5. ACD activates proinflammatory MAPK signaling following sequestration of bulk actin. (A, B) Representative Western blot (A) and quantification (B) of actin cross-linking in T84 cells inoculated with the strain of $V$. cholerae expressing MARTX $V_{c}$ toxin containing only active ACD. $n=3$ independent experiments. Data are reported as means \pm s.d. $(* * \mathrm{p}<0.01$, $* * * \mathrm{p}<0.001$, One-way ANOVA with Tukey's multiple comparison's test between indicated timepoints and $0 \%$ actin cross-linking from $0 \mathrm{~min}$ control). (C-E) Fold change and (F-H) representative Western blots of phosphorylated ERK (p-ERK, C and F), phosphorylated p38 (p-p38, D and G), and phosphorylated JNK (pJNK, E and H). Data are reported as means \pm s.d. (\#\# p<0.01, \#\#\# p<0.001, \#\#\#\# p<0.0001, One-way ANOVA with Tukey's multiple comparison's test between phosphorylated ERK, p38, or JNK at the indicated timepoints and a 0 min control normalized to a fold change of 1 . $n=4$ (C), 2 (D), or 3 (E) independent experiments. A third biological replicate for p-p38 quantification, but the active ACD only strain induced a stochastically high 273 -fold change compared to 0 min control (Fig. S6AB). 
A

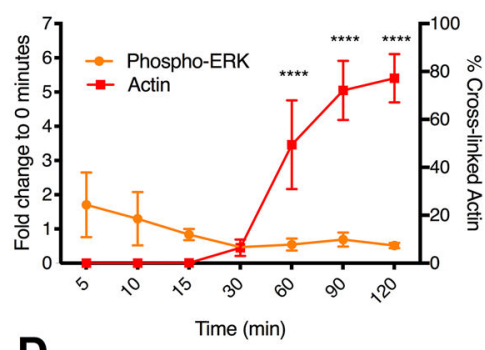

D

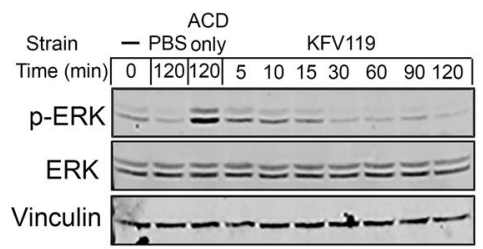

G

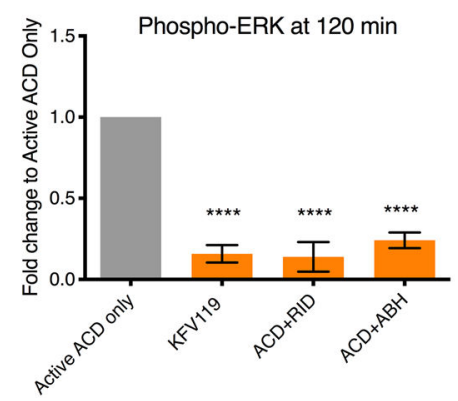

B

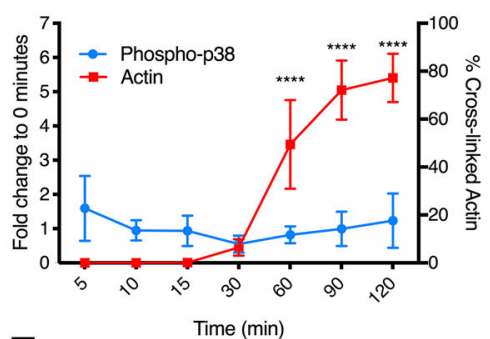

E

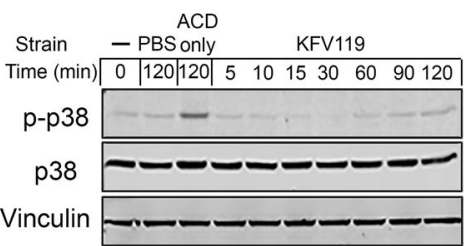

H

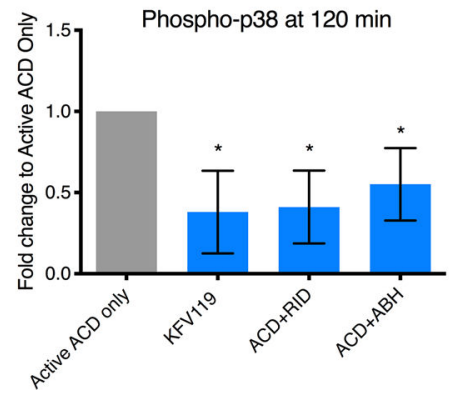

C

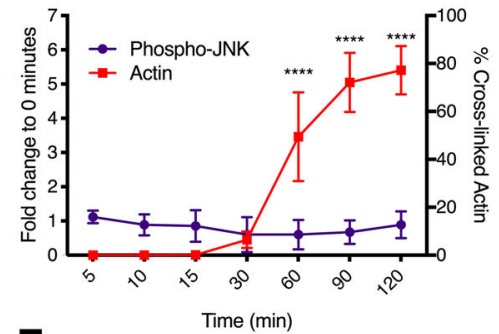

F

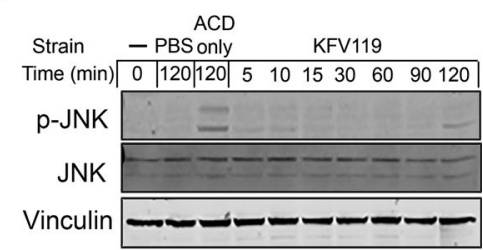

I

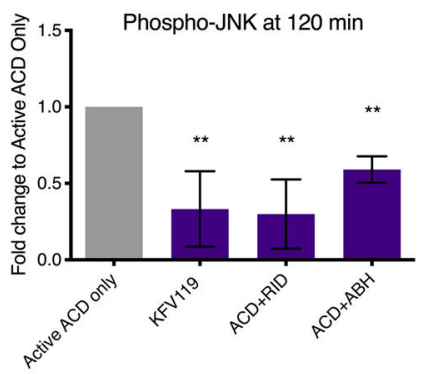

Fig. 6. RID and ABH block MAPK signaling prior to host detection to ACD-induced sequestration of bulk actin.

(A-C) Fold change and (D-F) representative Western blots of phosphorylated ERK (p-ERK, A and D), p38 (p-p38, B and E), or JNK (pJNK, C and F) in cells treated with $V$. cholerae KFV119. All samples were assayed from the same experiment so actin cross-linking is duplicated across panels. Data from densitometry of $n=3$ independent experiments reported as the mean \pm s.d. (****p<0.0001, One-way ANOVA with Tukey's multiple comparison's test between indicated timepoints and $0 \%$ actin cross-linking from 0 min control). (G-I) Quantification of fold change in phosphorylated ERK (p-ERK, G), phosphorylated p38 (pp38, H), and phosphorylated JNK (p-JNK, I) in cells treated with KFV119 V. cholerae parental strain or variants expressing the indicated active MARTX $_{V_{c}}$ toxin effectors compared to the strain expressing only active ACD at 120 minutes post-inoculation. Data from densitometry of $n=3$ independent experiments reported as the mean \pm s.d. $(* \mathrm{p}<0.05$, $* * \mathrm{p}<0.01, * * * * \mathrm{p}<0.0005$, Student's $t$-tests compared to Active ACD only strain). No statistically significant difference was observed between KFV119, ACD+RID Active, or $\mathrm{ACD}+\mathrm{ABH}$ Active strains compared to each other. 
A

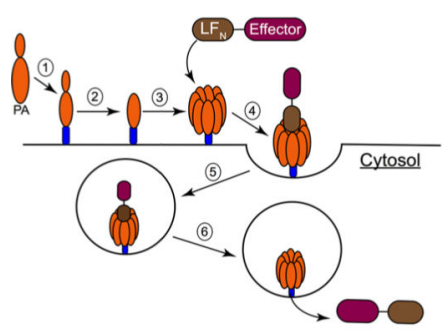

D

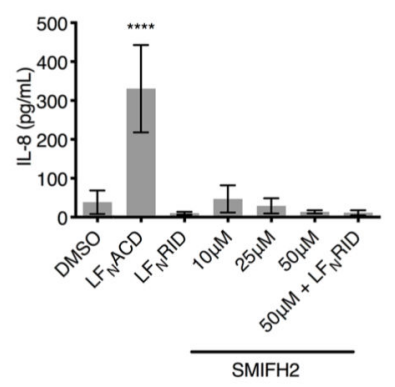

B

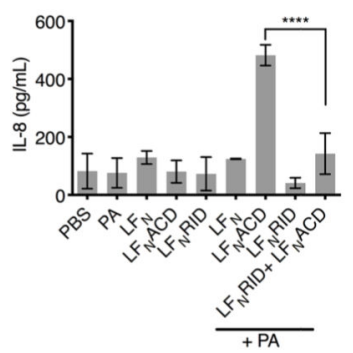

E

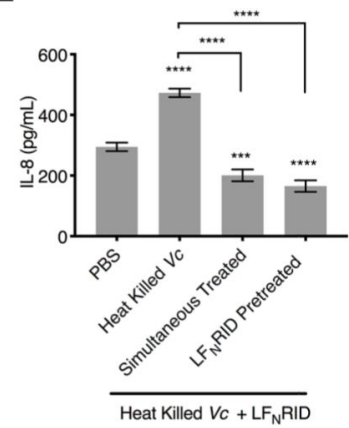

C
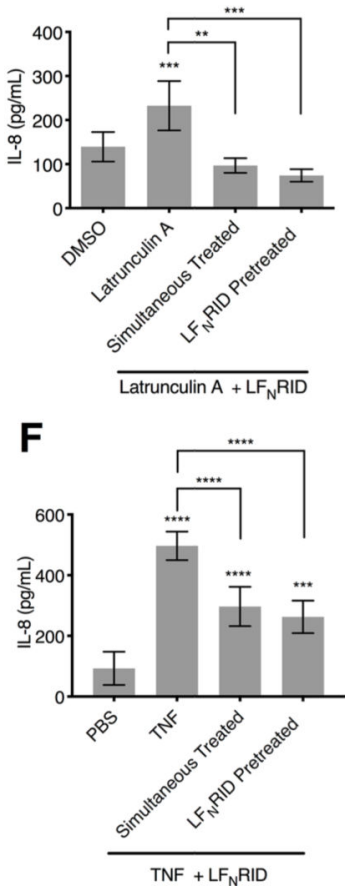

Fig. 7. Cytoskeletal collapse, and not formation of toxic actin oligomers, activates proinflammatory response in IECs.

(A) Schematic of intoxication with $\mathrm{LF}_{\mathrm{N}^{-}}$Effector fusion proteins. After PA binds to the anthrax toxin receptor (1) it is processed into its $63 \mathrm{kDa}$ active form $\left(\mathrm{PA}_{63}, 2\right)$, which oligomerizes to form a heptameric complex (3). The $\mathrm{LF}_{\mathrm{N}}$ domain of the $\mathrm{LF}_{\mathrm{N}}$-MARTX effector fusion binds to the $\mathrm{PA}_{63}$ heptamer (4), and he $\mathrm{LF}_{\mathrm{N}}$-Effector + PA63 complex enters the cell through receptor -mediated endocytosis (5). Acidification of the vacuole promotes the $\mathrm{PA}_{63}$ heptamer to form a pore in the vacuole membrane, releasing the $\mathrm{LF}_{\mathrm{N}}$-Effector fusion protein into the cytosol (6). (B) Quantification of IL-8 secretion measured from T84 IECs treated with $\mathrm{LF}_{\mathrm{N}}$ alone, $\mathrm{LF}_{\mathrm{N}} \mathrm{ACD}$, or $\mathrm{LF}_{\mathrm{N}} \mathrm{RID}$ in the presence or absence of PA. Data are from $n=3$ biological replicates reported as means \pm s.d. ( $* * * * p<0.0001$, One-way ANOVA with Tukey's multiple comparisons). (C, D) Quantification of IL-8 measured from T84 cells treated with latrunculin A (C) or SMIFH2 (D) alone or in combination with $\mathrm{LF}_{\mathrm{N}} \mathrm{RID}$ in the presence of PA as indicated. Data represent $n=6$ biological replicates reported as means \pm s.d. $(* * * \mathrm{p}<0.001, * * * * \mathrm{p}<0.0001$, One-way ANOVA with Tukey's multiple comparison's test). (E, F) Quantification of IL-8 measured from T84 cells treated with heat-killed $V$. cholerae $(V c)(\mathrm{E})$ or TNF $(\mathrm{F})$ in combination with $\mathrm{LF}_{\mathrm{N}} \mathrm{RID}$ in the presence of PA as indicated. Data from $n=3(\mathrm{E})$ or $n=6(\mathrm{~F})$ biological replicates reported as means \pm s.d. $(* * \mathrm{p}<0.01 * * * \mathrm{p}<0.001, * * * * \mathrm{p}<0.0001$, One-way ANOVA with Tukey's multiple comparison's test compared to PBS only or for samples as indicated). 


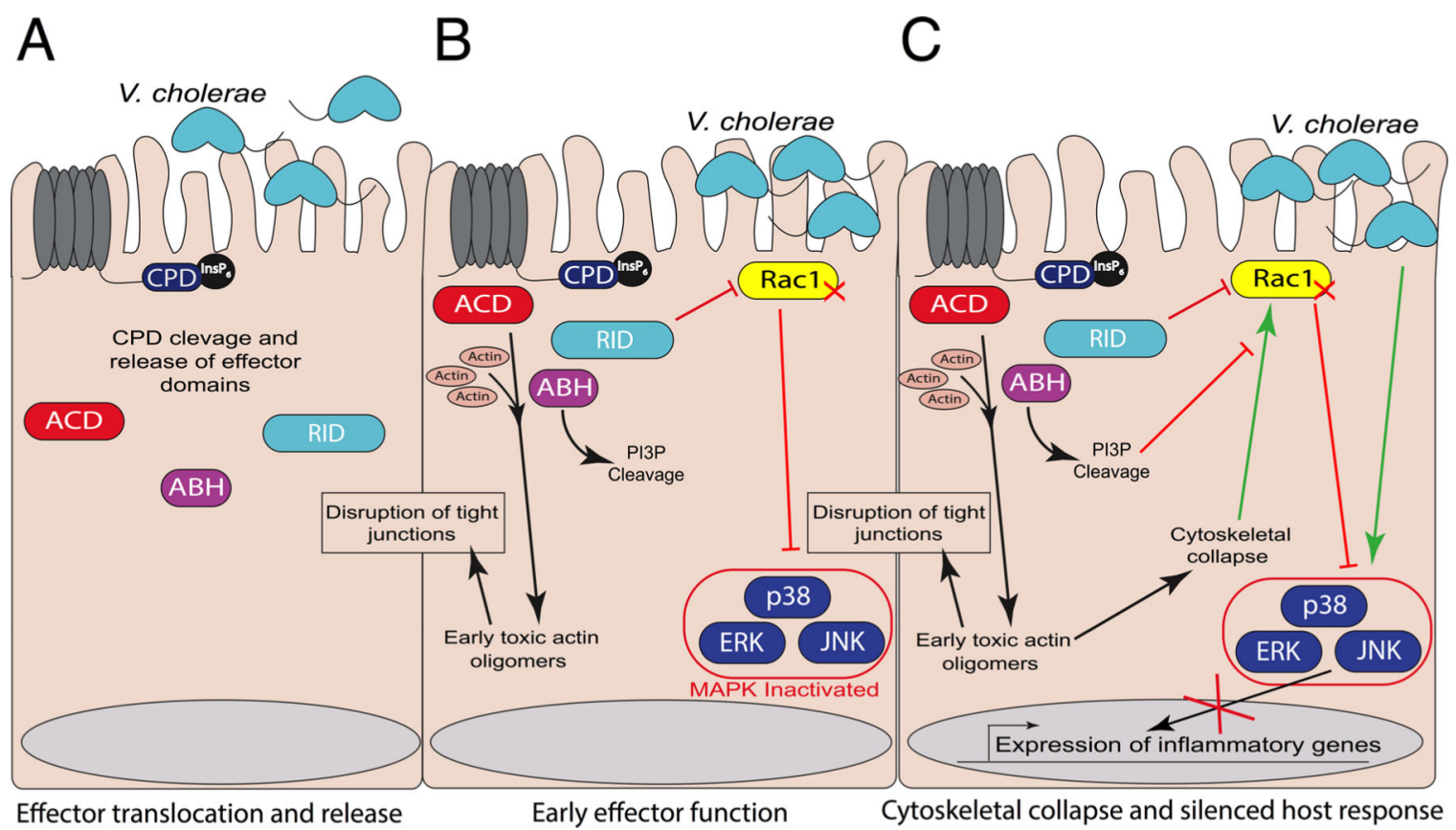

Fig. 8. Model of interplay between the multiple functions of the MARTX $\mathbf{V}_{c}$ toxin.

(A) After the MARTX $_{V c}$ toxin inserts into the plasma membrane of intestinal epithelial cells, the effector domains are separated from the holotoxin. (B) During the early stages of effector activity, ACD-mediated actin cross-linking forms toxic actin oligomers that disrupt tight junctions, compromising the intestinal barrier. ABH cleaves PI3P, and RID inactivates Rac1, thus blocking activation of MAPK signaling pathways. (C). ACD activity eventually leads to the sequestration of bulk actin and cytoskeletal collapse. Inactivation of Rac1 and the signaling pathways upstream of it prevent MAPK activation, leading to silencing of the inflammatory response to ACD-mediated destruction of the cytoskeleton and preventing host detection of bacterial PAMPs. 